Nicolas Charalambakis · François Murat

\title{
Approximation by finite elements, existence and uniqueness for a model of stratified thermoviscoplastic materials
}

Received: August 8, 2006 / Accepted: September 18, 2006

(c) 2006 The Autors ${ }^{1}$

Abstract In the present paper we consider for $a<x<b, 0<t<T$, the system of partial differential equations

$$
\begin{aligned}
& \rho(x) \frac{\partial v}{\partial t}-\frac{\partial}{\partial x}\left(\mu(x, \theta) \frac{\partial v}{\partial x}\right)=f, \\
& c(x, \theta) \frac{\partial \theta}{\partial t}=\mu(x, \theta)\left(\frac{\partial v}{\partial x}\right)^{2},
\end{aligned}
$$

completed by boundary conditions on $v$ and by initial conditions on $v$ and $\theta$. The unknowns are the velocity $v$ and the temperature $\theta$, while the coefficients $\rho, \mu$ and $c$ are Carathéodory functions which satisfy

$$
\begin{gathered}
0<c_{1} \leq \mu(x, s) \leq c_{2}, \quad \frac{\partial \mu}{\partial s}(x, s) \leq 0, \\
0<c_{3} \leq c(x, s) \leq c_{4}, \quad 0<c_{5} \leq \rho(x) \leq c_{6} .
\end{gathered}
$$

Communicated by the Editor-in-Chief

N. Charalambakis

Department of Civil Engineering, Aristotle University, GR 54124 Thessaloniki, Greece E-mail: charalam@ @ivil.auth.gr

F. Murat

Laboratoire Jacques-Louis Lions, Université Pierre et Marie Curie (Paris VI), Boîte courrier 187, 75252 Paris Cedex 05, France

E-mail:murat@ann.jussieu.fr

1 This is a "Springer Open Choice" article. Unrestricted non-commercial use, distribution, and reproduction in any medium is permitted, provided the original author and source are credited. 
This one dimensional system is a model for the behaviour of nonhomogeneous, stratified, thermoviscoplastic materials exhibiting thermal softening and temperature dependent rate of plastic work converted into heat. Under the above hypotheses we prove the existence of a solution by proving the convergence of a finite element approximation. Assuming further that $\mu$ is Lipschitz continuous in $s$, we prove the uniqueness of the solution, as well as its continuous dependence with respect to the data. We also prove its regularity when suitable hypotheses are made on the data. These results ensure the existence and uniqueness of one solution of the system in a class where the velocity $v$, the temperature $\theta$ and the stress $\sigma=\mu(x, \theta) \frac{\partial v}{\partial x}$ belong to $L^{\infty}((0, T) \times(a, b))$.

Keywords Thermoviscoplastic materials $\cdot$ nonhomogeneous materials $\cdot$ thermal softening · existence · uniqueness · Galerkin's method

Mathematics Subject Classification (2000) 74H20 - 74H25 - 65M60 - 35D05 . 35D10 $\cdot 35 \mathrm{R} 05 \cdot 74 \mathrm{C} 10 \cdot 74 \mathrm{~F} 05 \cdot 35 \mathrm{Q} 72 \cdot 35 \mathrm{M} 20$

\section{Contents}

Abstract ............................................... 171

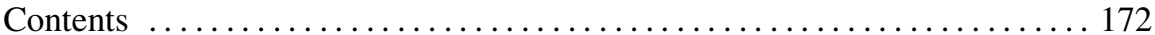

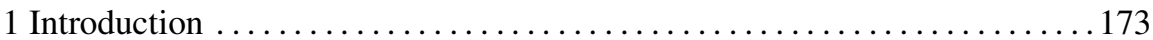

2 Approximation by finite elements and existence of a solution ......... 176

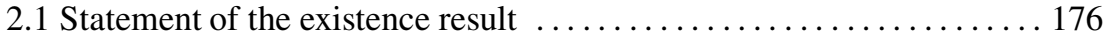

Theorem 2.1 (Existence) ............................. 177

2.1bis Existence results for Neumann and mixed boundary conditions . . 178 Theorem 2.1bis (Existence for Neumann boundary conditions) . . . . . 179

Theorem 2.1ter (Existence for mixed boundary conditions) ........ 180

2.2 Transformation of the problem by a change of unknown functions ...180

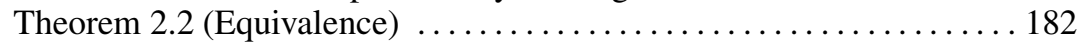

Theorem 2.3 (Existence for the transformed system) $\ldots \ldots \ldots \ldots \ldots 183$

2.3 Definition of the approximating problem $\ldots \ldots \ldots \ldots \ldots \ldots \ldots \ldots \ldots . \ldots \ldots$

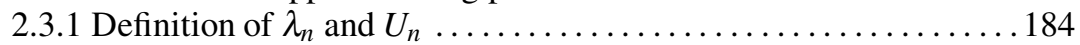

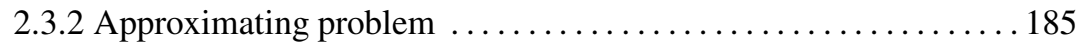

2.3.3 Local existence of the approximating solution ............ 186

2.4 Convergence of the approximating solution $\ldots \ldots \ldots \ldots \ldots \ldots \ldots . \ldots 186$

Theorem 2.4 (Convergence of Galerkin's approximation) ......... 186

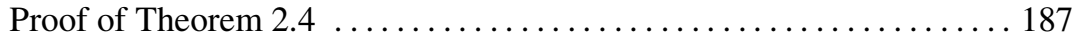

First step: a priori estimates ........................... 187

Second step: study of the approximating stress $\ldots \ldots \ldots \ldots \ldots \ldots \ldots 189$

Third step: strong convergence of $\tau_{n}$ in $L^{1}(Q) \ldots \ldots \ldots \ldots \ldots \ldots 192$

Fourth step: strong convergence of $\frac{\partial u_{n}}{\partial x}$ in $L^{2}(Q) \ldots \ldots \ldots \ldots \ldots . \ldots 193$

Fifth step: end of the proof of Theorem $2.4 \ldots \ldots \ldots \ldots \ldots \ldots \ldots$

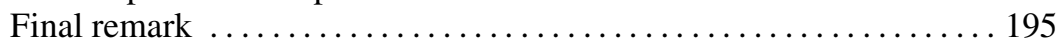

3 Uniqueness, continuity with respect to the data and regularity

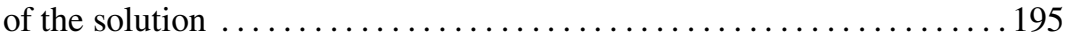

3.1 Statement of the uniqueness and continuity result ............. 196

Theorem 3.1 (Uniqueness and continuity with respect to the data) ... 196 
3.2 A straightforward regularity result for $\tau$................... 196

Proposition 3.1 (Straightforward regularity for $\tau$ ) .............. 197

Proof of Proposition 3.1 .............................. 197

3.3 Proof of the uniqueness and continuity Theorem $3.1 \ldots \ldots \ldots \ldots . \ldots 198$

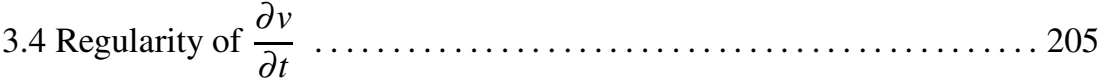

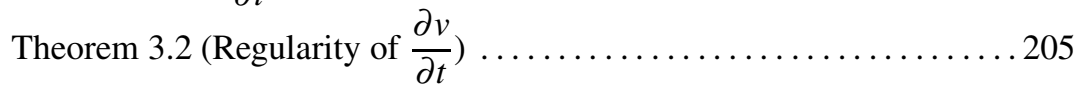

Proof of Theorem 3.2 ................................ 207

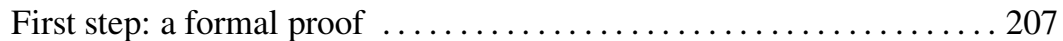

Second step: an abstract result $\ldots \ldots \ldots \ldots \ldots \ldots \ldots \ldots \ldots \ldots . \ldots 208$

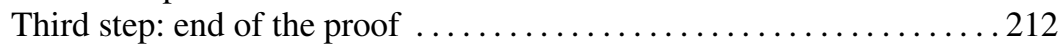

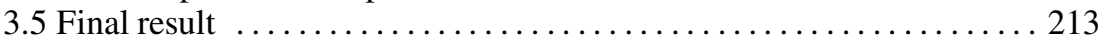

Theorem 3.3 (Existence, uniqueness and regularity) .............2 213

Proof of Theorem 3.3 ................................ 215

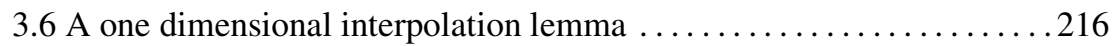

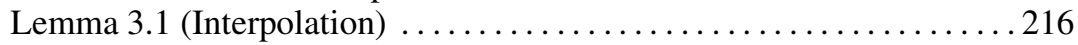

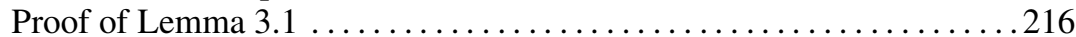

Acknowledgments ............................................ 217

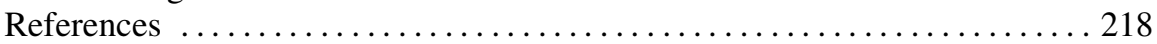

\section{Introduction}

The present paper deals with a system of two partial differential equations describing the shearing of a nonhomogeneous, stratified, thermoviscoplastic material exhibiting thermal softening.

After the pioneering paper of Dafermos and Hsiao [4] studying the adiabatic shearing of a newtonian fluid with temperature dependent viscosity, many papers have been devoted to the study of the existence and asymptotic stability in time of the solutions of this problem for various classes of materials (see, for instance, the paper [1] of one of us and the papers [9] and [10] of Tzavaras). The problem of shear stability is related to the understanding of the emergence and development of shear bands, and recent references on physical and engineering aspects of it can be found, for example, in the book of Wright [11]. However, in all these papers, the material is supposed to be homogeneous, in the sense that the viscosity function, the heat coefficient and the referential density are supposed to depend only on the temperature.

Here, the material is supposed to be nonhomogeneous in the sense that the viscosity function, the heat coefficient and the referential density depend (not necessarily smoothly) on the space variable. Indeed the material under consideration is stratified in the sense that it is made of layers perpendicular to a given direction of space, say the $x$ direction. Assuming that the material is sheared uniformly in a direction perpendicular to $x$ between two parallel planes located at say $x=a$ and $x=b$, and disregarding the effect of the other boundary conditions, this allows one to reduce to one dimensional (in space) partial differential equations.

Assuming that the elastic strain is equal to zero, the balance laws of the process which relate the three unknows of the problem, namely the velocity $v(t, x)$ in the direction of the shearing, the shear stress $\sigma(t, x)$ in the same direction and 
the temperature $\theta(t, x)$, lead to the following system of two partial differential equations

$$
\begin{array}{ll}
\rho(x) \frac{\partial v}{\partial t}=\frac{\partial \sigma}{\partial x}+f(t, x) & \text { in }(0, T) \times(a, b), \\
c(x, \theta) \frac{\partial \theta}{\partial t}=\sigma \frac{\partial v}{\partial x} & \text { in }(0, T) \times(a, b),
\end{array}
$$

where $T>0$ is given. In this system, $f(t, x), \rho(x)$ and $c(x, \theta)$ are given: $f(t, x)$ denotes the external (body) forces acting on the material, $\rho(x)$ the referential density, which is supposed to depend only on $x$, and $c(x, \theta)$ the heat coefficient, which is given by

$$
c(x, \theta)=\frac{1}{\beta(x, \theta)} \rho(x) h(x, \theta),
$$

where $h(x, \theta)$, the specific heat, and $\beta(x, \theta)$, the rate of plastic work converted into heat, are given functions which can depend both on $x$ and on the temperature. In almost all works concerned with this problem under adiabatic conditions, $\beta$ is supposed to be a constant which is equal to 0.9. However recent experimental work of Hodowany et al. [5] based on a Kolski bar technique, and theoretical studies by Rosakis et al. [8] suggest that $\beta$ can depend, among others, on the temperature. We actually prove in our paper [3] that homogenization of nonhomogeneous, stratified, thermoviscoplastic materials where $c$ depends only on $x$ produces an homogenized material where $c$ in general does depend on the temperature.

In the present paper, the shear stress $\sigma(t, x)$ is supposed to be given by the constitutive law

$$
\sigma=\mu(x, \theta) \frac{\partial v}{\partial x} \text { in }(0, T) \times(a, b),
$$

where the viscosity function $\mu$ is a given function of $x$ and of the temperature which satisfies

$$
\frac{\partial \mu}{\partial s}(x, s) \leq 0
$$

this inequality expresses the thermal softening of the material. This law is a limit case of the rheological constitutive law (see the paper [7] of Molinari and Clifton)

$$
\sigma=\mu(x, \theta, \gamma)\left|\frac{\partial v}{\partial x}\right|^{n-1} \frac{\partial v}{\partial x}
$$

(where $0<n<1$ ) which describes a thermoviscoplastic material exhibiting strain dependence, thermal softening and strain rate sensitivity. In the present limit case, the strain rate sensitivity $n$ is equal to 1 . On the other hand, the strain $\gamma$ acts as a hidden variable driven by the compatibility equation $\frac{\partial v}{\partial x}=\frac{\partial \gamma}{\partial t}$; but under slowly varying shear stress, it was observed that the strain $\gamma$ is quasi proportional to the temperature $\theta$. It was also observed in experiments at different temperature levels that the linear law is justified between certain values of the stress (see the book of Lemaitre and Chaboche [6]). The fact that we will prove in the present paper that the stress is bounded therefore justifies in part the use of the limit case $n=1$. However, from the mathematical point of view, the method used in the present 
paper should be modified in the general case where $n<1$ and where the strain $\gamma$ is taken into consideration.

The shearing is caused by time dependent imposed boundary velocities at $x=a$ and $x=b$, namely

$$
v(t, a)=v_{a}(t), \quad v(t, b)=v_{b}(t) \quad \text { in }(0, T),
$$

where $v_{a}$ and $v_{b}$ are given. We will also consider the case where the shearing is caused by time dependent shears imposed at $x=a$ and $x=b$, namely

$$
\sigma(t, a)=\sigma_{a}(t), \quad \sigma(t, b)=\sigma_{b}(t) \quad \text { in }(0, T),
$$

where $\sigma_{a}$ and $\sigma_{b}$ are given, and the case where the velocity is imposed on one boundary, say $x=a$, and the shear on the other one, namely

$$
v(t, a)=v_{a}(t), \quad \sigma(t, b)=\sigma_{b}(t) \quad \text { in }(0, T) .
$$

Finally the system is complemented by initial conditions on the velocity and temperature, namely

$$
v(0, x)=v_{0}(x), \quad \theta(0, x)=\theta_{0}(x) \quad \text { in }(a, b) .
$$

In short, when the shearing is caused by imposed boundary velocities, the thermomecanical process under consideration is described by the following initial boundary value system

$$
\begin{gathered}
\rho(x) \frac{\partial v}{\partial t}-\frac{\partial}{\partial x}\left(\mu(x, \theta) \frac{\partial v}{\partial x}\right)=f(t, x) \quad \text { in }(0, T) \times(a, b), \\
c(x, \theta) \frac{\partial \theta}{\partial t}=\mu(x, \theta)\left(\frac{\partial v}{\partial x}\right)^{2} \quad \text { in }(0, T) \times(a, b), \\
v(t, a)=v_{a}(t), \quad v(t, b)=v_{b}(t) \quad \text { in }(0, T), \\
v(0, x)=v_{0}(x) \quad \text { in }(a, b), \\
\theta(0, x)=\theta_{0}(x) \quad \text { in }(a, b),
\end{gathered}
$$

where $v(t, x)$ and $\theta(t, x)$ are unknown, while $\rho, \mu, c, f, v_{a}, v_{b}, v_{0}$ and $\theta_{0}$ are given; as said above, the stress $\sigma(t, x)$ is given by

$$
\sigma=\mu(x, \theta) \frac{\partial v}{\partial x} \quad \text { in }(0, T) \times(a, b) .
$$

The goal of the present paper is to prove the existence and uniqueness of the solution of this system, as well as its continuous dependence with respect to the data and some regularity properties. Using Schauder's fixed point theorem, we proved in [2] an existence result for (1.1)-(1.5) under homogeneous boundary conditions. In Section 2 of the present paper, we will prove the existence of solutions by using a $\mathbb{P}^{1}$ (in $v$ ) and $\mathbb{P}^{0}$ (in $\theta$ ) finite elements approximation of this problem. This method has the avantage to be more constructive and to prove the convergence of an approximating scheme (see Subsection 2.4). Actually, before of proving this existence result, we will first perform a transformation of the problem by introducing new unknown functions (see Subsection 2.2). This transformation will be 
used in the whole of the present paper. In Section 3 we will prove the uniqueness of the solution of (1.1)-(1.5), as well as its local Lipschitz continuous dependence with respect to the data and some regularity results. Combining all those results ensures the existence and uniqueness of the solution of (1.1)-(1.5) in a class where velocity, temperature and stress belong to $L^{\infty}((0, T) \times(a, b))$ (see Theorem 3.3).

These existence, uniqueness, continuity with respect to the data and regularity results continue to hold, with the same proofs, in the case where the boundary conditions (1.3) are replaced by Neumann boundary conditions

$$
\sigma(t, a)=\sigma_{a}(t), \quad \sigma(t, b)=\sigma_{b}(t) \quad \text { in }(0, T),
$$

or by mixed boundary conditions

$$
v(t, a)=v_{a}(t), \quad \sigma(t, b)=\sigma_{b}(t) \quad \text { in }(0, T),
$$

(see Subsection 2.1bis and the comments after Theorems 3.1, 3.2 and 3.3).

In our paper [3], we investigate the asymptotic behaviour of a thermoviscoplastic material made of numerous layers of different phases of the type considered here, each of those layers having a small thickness, i.e. the homogenization of such materials, and we prove that system (1.1)-(1.5) is stable by homogenization.

Finally, we have to stress the importance in the proofs of the one dimensionality in space of the partial differential equations; in several dimensions most of the results given here should be very different and are beyond our reach.

\section{Approximation by finite elements and existence of a solution}

This Section presents an existence result for the initial boundary value system (1.1)-(1.5). The proof which is given here uses Galerkin's method for $\mathbb{P}^{1} / \mathbb{P}^{0}$ finite elements approximation. Another proof using Schauder's fixed point theorem was given in our paper [2]. The proof presented here, even if technically a little bit more complicated, has the advantage to provide the convergence of an approximating scheme of system (1.1)-(1.5).

We state this existence result in Subsection 2.1. Its proof will follow from a change of unknowns functions that we will perform in Subsection 2.2, passing from the unknowns $(v, \theta)$ to the equivalent unknowns $(u, \tau)$. This transformation will be used in the whole of the present paper. The existence result for the unknowns $(u, \tau)$ and the convergence of the Galerkin's method will be proved in Subsection 2.4.

\subsection{Statement of the existence result}

In the whole of the present paper, we consider $a, b$ and $T$ in $\mathbb{R}$ with $a<b$ and $T>0$ and we set

$$
(a, b)=\Omega, \quad(0, T) \times(a, b)=Q .
$$

We assume that the fonctions $\mu: \Omega \times \mathbb{R} \rightarrow \mathbb{R}$ and $c: \Omega \times \mathbb{R} \rightarrow \mathbb{R}$ are Carathéodory functions which are uniformly (in $x$ ) continuous in $s$, i.e.

$$
\left\{\begin{array}{l}
x \rightarrow \mu(x, s) \text { is measurable } \quad \forall s \in \mathbb{R}, \\
\left|\mu(x, s)-\mu\left(x, s^{\prime}\right)\right| \leq \omega\left(s,\left|s-s^{\prime}\right|\right) \quad \text { a.e. } x \in \Omega, \forall s, s^{\prime} \in \mathbb{R},
\end{array}\right.
$$




$$
\left\{\begin{array}{l}
x \rightarrow c(x, s) \text { is measurable } \quad \forall s \in \mathbb{R}, \\
\left|c(x, s)-c\left(x, s^{\prime}\right)\right| \leq \omega\left(s,\left|s-s^{\prime}\right|\right) \quad \text { a.e. } x \in \Omega, \forall s, s^{\prime} \in \mathbb{R},
\end{array}\right.
$$

where $\omega(s, \hat{s})$ is a modulus of continuity, i.e.

$$
\omega(s, \hat{s}) \rightarrow 0 \quad \text { when } \quad \hat{s} \rightarrow 0, \quad \forall s \in \mathbb{R},
$$

and that

$$
\rho \in L^{\infty}(\Omega)
$$

We also assume that the functions $\mu, c$ and $\rho$ satisfy

$$
\begin{array}{cc}
c_{1} \leq \mu(x, s) \leq c_{2} & \text { a.e. } x \in \Omega, \forall s \in \mathbb{R}, \\
c_{3} \leq c(x, s) \leq c_{4} & \text { a.e. } x \in \Omega, \forall s \in \mathbb{R}, \\
c_{5} \leq \rho(x) \leq c_{6} & \text { a.e. } x \in \Omega,
\end{array}
$$

where $0<c_{1} \leq c_{2}<+\infty, 0<c_{3} \leq c_{4}<+\infty$ and $0<c_{5} \leq c_{6}<+\infty$ are constants. Moreover we assume in this Section (this hypothesis will not be made in Subsections 3.1-3.4 below) that $\mu$ is nonincreasing in $s$, i.e.

$$
\frac{\partial \mu}{\partial s}(x, s) \leq 0 \quad \text { in } \mathscr{D}^{\prime}(\Omega \times \mathbb{R}) .
$$

For what concerns the external forces and the initial and boundary conditions, we assume in this Section that

$$
\begin{gathered}
f \in L^{2}\left(0, T ; L^{2}(\Omega)\right), \\
v_{a} \in H^{1}(0, T), \quad v_{b} \in H^{1}(0, T), \\
v_{0} \in H^{1}(\Omega), \\
\theta_{0} \in L^{1}(\Omega),
\end{gathered}
$$

and that the following compatibility condition holds true

$$
v_{0}(a)=v_{a}(0), \quad v_{0}(b)=v_{b}(0) .
$$

We then have the following existence result.

Theorem 2.1 (Existence) Assume that hypotheses (2.1)-(2.12) hold true. Then there exists at least one couple $(v, \theta)$ which satisfies (1.1)-(1.5) in the following sense

$$
\begin{gathered}
v \in L^{\infty}\left(0, T ; H^{1}(\Omega)\right) \cap H^{1}\left(0, T ; L^{2}(\Omega)\right), \\
\theta \in W^{1,1}\left(0, T ; L^{1}(\Omega)\right), \\
\rho(x) \frac{\partial v}{\partial t}-\frac{\partial}{\partial x}\left(\mu(x, \theta) \frac{\partial v}{\partial x}\right)=f \quad \text { in } \mathscr{D}^{\prime}(Q), \\
c(x, \theta) \frac{\partial \theta}{\partial t}=\mu(x, \theta)\left(\frac{\partial v}{\partial x}\right)^{2} \quad \text { in } \mathscr{D}^{\prime}(Q), \\
v(t, a)=v_{a}(t), \quad v(t, b)=v_{b}(t) \quad \text { a.e. } t \in(0, T),
\end{gathered}
$$




$$
\begin{array}{ll}
v(0, x)=v_{0}(x) & \text { a.e. } x \in \Omega, \\
\theta(0, x)=\theta_{0}(x) & \text { a.e. } x \in \Omega .
\end{array}
$$

Moreover the stress $\sigma$ defined by (1.6), namely

$$
\sigma=\mu(x, \theta) \frac{\partial v}{\partial x}
$$

satisfies

$$
\sigma \in L^{\infty}\left(0, T ; L^{2}(\Omega)\right) \cap L^{2}\left(0, T ; H^{1}(\Omega)\right) .
$$

Finally for this solution, the following a priori estimates hold true

$$
\begin{gathered}
\|v\|_{L^{\infty}\left(0, T ; H^{1}(\Omega)\right)}+\left\|\frac{\partial v}{\partial t}\right\|_{L^{2}\left(0, T ; L^{2}(\Omega)\right)} \leq C^{\star}, \\
\|\theta\|_{L^{\infty}\left(0, T ; L^{1}(\Omega)\right)}+\left\|\frac{\partial \theta}{\partial t}\right\|_{L^{1}\left(0, T ; L^{1}(\Omega)\right)} \leq C^{\star}, \\
\|\sigma\|_{L^{\infty}\left(0, T ; L^{2}(\Omega)\right)}+\|\sigma\|_{L^{2}\left(0, T ; H^{1}(\Omega)\right)} \leq C^{\star},
\end{gathered}
$$

where $C^{\star}$ denotes a constant which depends only on $c_{1}, c_{2}, c_{3}, c_{4}, c_{5}, c_{6},(b-a)$ and $K$, when the data satisfy

$$
\left\{\begin{array}{l}
\|f\|_{L^{2}\left(0, T ; L^{2}(\Omega)\right)}+\left\|v_{a}\right\|_{H^{1}(0, T)}+\left\|v_{b}\right\|_{H^{1}(0, T)}+ \\
+\left\|v_{0}\right\|_{H^{1}(\Omega)}+\left\|\theta_{0}\right\|_{L^{1}(\Omega)} \leq K .
\end{array}\right.
$$

Note that every term of equations (2.15) and (2.16) has a meaning in the sense of distributions, since $\mu(x, \theta)$ belongs to $L^{\infty}(Q)$. Similarly the boundary conditions (2.17) have a meaning, since $v$ belongs to $L^{\infty}\left(0, T ; H^{1}(\Omega)\right)$ and since $H^{1}(\Omega) \subset C^{0}(\bar{\Omega})$ when $\Omega$ is one dimensional. Finally the initial conditions (2.18) and (2.19) have a meaning since $v$ belongs to $H^{1}\left(0, T ; L^{2}(\Omega)\right) \subset$ $\subset C^{0}\left([0, T] ; L^{2}(\Omega)\right)$ while $\theta$ belongs to $W^{1,1}\left(0, T ; L^{1}(\Omega)\right) \subset C^{0}\left([0, T] ; L^{1}(\Omega)\right)$.

The regularity (2.21) on the stress $\sigma$ is very specific to the one dimensionality of the problem. Indeed it immediately follows on one hand from (2.4), (2.13) and from the definition (2.20) of $\sigma$, and on the other hand from equation (2.15), which reads as $\frac{\partial \sigma}{\partial x}=\rho \frac{\partial v}{\partial t}-f$, and from (2.6), (2.13) and (2.8).

The a priori estimates (2.22)-(2.24) on $v, \theta$ and $\sigma$ of Theorem 2.1 are stated here only for the solution built in the proof below. Actually by the uniqueness Theorem 3.1, these a priori estimates hold true for every (actually for the unique) solution of (2.13)-(2.19) whenever hypothesis (3.1) is made.

\section{1bis Existence results for Neumann and mixed boundary conditions}

As said in the Introduction, the boundary conditions (1.3) on $v$ can be replaced by Neumann boundary conditions (1.3bis) on $\sigma$ or by mixed boundary conditions (1.3ter) on $v$ and $\sigma$. 
In the case of Neumann boundary conditions we assume that

$$
\sigma_{a} \in L^{2}(0, T), \quad \sigma_{b} \in L^{2}(0, T),
$$

and, in the case of mixed boundary conditions, that

$$
v_{a} \in H^{1}(0, T), \quad \sigma_{b} \in L^{2}(0, T),
$$

and that the compatibility condition

$$
v_{0}(a)=v_{a}(0)
$$

holds true.

We then have the two following existence results, which can be proved along the lines of the proof of Theorem 2.1.

Theorem 2.1bis (Existence for Neumann boundary conditions) Assume that hypotheses (2.1)-(2.8), (2.26), (2.10) and (2.11) hold true. Then there exists at least one couple $(v, \theta)$ which satisfies (1.1), (1.2), (1.3bis), (1.4) and (1.5) in the following sense

$$
\begin{aligned}
& v \in L^{\infty}\left(0, T ; H^{1}(\Omega)\right) \cap H^{1}\left(0, T ; L^{2}(\Omega)\right), \\
& \theta \in W^{1,1}\left(0, T ; L^{1}(\Omega)\right), \\
& \left\{\begin{array}{l}
\int_{\Omega} \rho(x) \frac{\partial v}{\partial t} w d x+\int_{\Omega} \mu(x, \theta) \frac{\partial v}{\partial x} \frac{\partial w}{\partial x} d x= \\
=\int_{\Omega} f w d x+\sigma_{b}(t) w(b)-\sigma_{a}(t) w(a) \text { in } \mathscr{D}^{\prime}(0, T), \\
\forall w \in H^{1}(\Omega),
\end{array}\right. \\
& c(x, \theta) \frac{\partial \theta}{\partial t}=\mu(x, \theta)\left(\frac{\partial v}{\partial x}\right)^{2} \quad \text { in } \mathscr{D}^{\prime}(Q), \\
& v(0, x)=v_{0}(x) \quad \text { a.e. } x \in \Omega \text {, } \\
& \theta(0, x)=\theta_{0}(x) \quad \text { a.e. } x \in \Omega \text {. }
\end{aligned}
$$

Moreover the stress $\sigma$ defined by (1.6), namely $\sigma=\mu(x, \theta) \frac{\partial v}{\partial x}$, satisfies

$$
\sigma \in L^{\infty}\left(0, T ; L^{2}(\Omega)\right) \cap L^{2}\left(0, T ; H^{1}(\Omega)\right) .
$$

Finally for this solution, the following a priori estimates hold true

$$
\begin{gathered}
\|v\|_{L^{\infty}\left(0, T ; H^{1}(\Omega)\right)}+\left\|\frac{\partial v}{\partial t}\right\|_{L^{2}\left(0, T ; L^{2}(\Omega)\right)} \leq C_{\mathrm{bis}}^{\star} \\
\|\theta\|_{L^{\infty}\left(0, T ; L^{1}(\Omega)\right)}+\left\|\frac{\partial \theta}{\partial t}\right\|_{L^{1}\left(0, T ; L^{1}(\Omega)\right)} \leq C_{\mathrm{bis}}^{\star} \\
\|\sigma\|_{L^{\infty}\left(0, T ; L^{2}(\Omega)\right)}+\|\sigma\|_{L^{2}\left(0, T ; H^{1}(\Omega)\right)} \leq C_{\mathrm{bis}}^{\star}
\end{gathered}
$$


where $C_{\mathrm{bis}}^{\star}$ denotes a constant which depends only on $c_{1}, c_{2}, c_{3}, c_{4}, c_{5}, c_{6},(b-a)$ and $K_{\text {bis }}$, when the data satisfy

$$
\left\{\begin{array}{l}
\|f\|_{L^{2}\left(0, T ; L^{2}(\Omega)\right)}+\left\|\sigma_{a}\right\|_{L^{2}(0, T)}+\left\|\sigma_{b}\right\|_{L^{2}(0, T)}+ \\
+\left\|v_{0}\right\|_{H^{1}(\Omega)}+\left\|\theta_{0}\right\|_{L^{1}(\Omega)} \leq K_{\mathrm{bis}} .
\end{array}\right.
$$

As usual in Neumann's problem, both the equation (1.1) (in the sense of $\mathscr{D}^{\prime}(Q)$ ) and the boundary conditions (1.3bis) (which here have a meaning since $\sigma$ belongs to $L^{2}\left(0, T ; H^{1}(\Omega)\right)$ and $\left.H^{1}(\Omega) \subset C^{0}(\bar{\Omega})\right)$, are contained in the variational formulation (2.31).

Theorem 2.1ter (Existence for mixed boundary conditions) Assume that hypotheses (2.1)-(2.8), (2.27), (2.28), (2.10) and (2.11) hold true. Then there exists at least one couple $(v, \theta)$ which satisfies (1.1), (1.2), (1.3ter), (1.4) and (1.5), in the sense that (2.29), (2.30),

$$
\left\{\begin{array}{l}
\int_{\Omega} \rho(x) \frac{\partial v}{\partial t} w d x+\int_{\Omega} \mu(x, \theta) \frac{\partial v}{\partial x} \frac{\partial w}{\partial x} d x= \\
=\int_{\Omega} f w d x+\sigma_{b}(t) w(b) \quad \text { in } \mathscr{D}^{\prime}(0, T), \\
\forall w \in H^{1}(\Omega) \quad \text { with } w(a)=0, \\
v(a, t)=v_{a}(t) \quad \text { a.e. } t \in(0, T),
\end{array}\right.
$$

(2.32), (2.33) and (2.34) hold true.

Moreover the stress $\sigma$ defined by (1.6), namely $\sigma=\mu(x, \theta) \frac{\partial v}{\partial x}$, satisfies (2.35).

Finally for this solution the a priori estimates (2.36)-(2.38) hold true with $C_{\mathrm{bis}}^{\star}$ replaced by $C_{\text {ter }}^{\star}$, where $C_{\text {ter }}^{\star}$ denotes a constant which depends only on $c_{1}, c_{2}, c_{3}$, $c_{4}, c_{5}, c_{6},(b-a)$ and $K_{\text {ter }}$, when the data satisfy

$$
\left\{\begin{array}{l}
\|f\|_{L^{2}\left(0, T ; L^{2}(\Omega)\right)}+\left\|v_{a}\right\|_{H^{1}(0, T)}+\left\|\sigma_{b}\right\|_{L^{2}(0, T)}+ \\
+\left\|v_{0}\right\|_{H^{1}(\Omega)}+\left\|\theta_{0}\right\|_{L^{1}(\Omega)} \leq K_{\mathrm{ter}} .
\end{array}\right.
$$

\subsection{Transformation of the problem by a change of unknown functions}

Actually it is more convenient to write the system (1.1)-(1.5) in an equivalent but different way. Let us describe formally this equivalent formulation.

We begin by a translation which reduces the problem to homogeneous boundary conditions. (Such a translation has not to be done when Neumann boundary conditions are concerned, and it has to be conveniently modified when mixed boundary conditions are concerned.) Define

$$
\bar{v}(t, x)=\frac{(x-a) v_{b}(t)+(b-x) v_{a}(t)}{b-a}
$$




$$
\begin{aligned}
u_{0}(x) & =v_{0}(x)-\bar{v}(0, x), \\
u(t, x) & =v(t, x)-\bar{v}(t, x) .
\end{aligned}
$$

Note that

$$
u_{0} \in H_{0}^{1}(\Omega)
$$

when $v_{0}, v_{a}$ and $v_{b}$ satisfy hypotheses (2.9), (2.10) and (2.12).

Then $(v, \theta)$ is a solution of (1.1)-(1.5) if and only if $(u, \theta)$ is a solution of

$$
\begin{gathered}
\rho(x) \frac{\partial u}{\partial t}-\frac{\partial}{\partial x}\left(\mu(x, \theta)\left(\frac{\partial u}{\partial x}+\frac{\partial \bar{v}}{\partial x}\right)\right)=f-\rho(x) \frac{\partial \bar{v}}{\partial t} \quad \text { in }(0, T) \times(a, b), \\
c(x, \theta) \frac{\partial \theta}{\partial t}=\mu(x, \theta)\left(\frac{\partial u}{\partial x}+\frac{\partial \bar{v}}{\partial x}\right)^{2} \quad \text { in }(0, T) \times(a, b), \\
u(t, a)=0, \quad u(t, b)=0 \quad \text { in }(0, T), \\
u(0, x)=u_{0}(x) \quad \text { in }(a, b), \\
\theta(0, x)=\theta_{0}(x) \quad \text { in }(a, b) .
\end{gathered}
$$

We will now perform a change of unknown function by replacing $\theta(t, x)$ by a new unknown $\tau(t, x)$. Define the function $M: \Omega \times \mathbb{R} \rightarrow \mathbb{R}$ by

$$
M(x, s)=\int_{\theta_{0}(x)}^{s} c\left(x, s^{\prime}\right) \mu\left(x, s^{\prime}\right) d s^{\prime} \quad \text { a.e. } x \in \Omega, \forall s \in \mathbb{R} .
$$

Since $0<c_{3} c_{1} \leq c\left(x, s^{\prime}\right) \mu\left(x, s^{\prime}\right) \leq c_{4} c_{2}<+\infty$, the function $s \rightarrow M(x, s)$ is oneto-one from $\mathbb{R}$ into $\mathbb{R}$ for almost every $x \in \Omega$. Let $N(x, r)$ denote its reciprocal function, defined by

$$
M(x, s)=r \quad \Longleftrightarrow \quad N(x, r)=s \quad \text { a.e. } x \in \Omega \text {. }
$$

Note that the functions $M: \Omega \times \mathbb{R} \rightarrow \mathbb{R}$ and $N: \Omega \times \mathbb{R} \rightarrow \mathbb{R}$ are Carathéodory functions, and that the functions $s \rightarrow M(x, s)$ and $r \rightarrow N(x, r)$ are strictly increasing, Lipschitz continuous functions which satisfy

$$
\begin{gathered}
\left\{\begin{aligned}
& c_{3} c_{1}\left(s-s^{\prime}\right) \leq M(x, s)-M\left(x, s^{\prime}\right) \leq c_{4} c_{2}\left(s-s^{\prime}\right) \\
& \text { a.e. } x \in \Omega, \forall s, s^{\prime} \in \mathbb{R}, s \geq s^{\prime},
\end{aligned}\right. \\
\left\{\begin{array}{c}
\frac{1}{c_{4} c_{2}}\left(r-r^{\prime}\right) \leq N(x, r)-N\left(x, r^{\prime}\right) \leq \frac{1}{c_{3} c_{1}}\left(r-r^{\prime}\right) \\
\text { a.e. } x \in \Omega, \forall r, r^{\prime} \in \mathbb{R}, r \geq r^{\prime}, \\
M\left(x, \theta_{0}(x)\right)=0, \\
N(x, 0)=\theta_{0}(x) .
\end{array}\right.
\end{gathered}
$$

Define now the new unknown $\tau(t, x)$ by

$$
\tau(t, x)=M(x, \theta(t, x)) .
$$


The reason for this definition is that multiplying equation (2.48) on $\theta$ by $\mu(x, \theta)$ (which is always finite and strictly positive), we see that (2.48) is equivalent to

$$
\frac{\partial \tau}{\partial t}=\left|\mu(x, \theta)\left(\frac{\partial u}{\partial x}+\frac{\partial \bar{v}}{\partial x}\right)\right|^{2} \quad \text { in }(0, T) \times(a, b),
$$

while the initial condition (2.51) is equivalent to

$$
\tau(0, x)=0 \quad \text { in }(a, b) .
$$

We finally define the function $\lambda: \Omega \times \mathbb{R} \rightarrow \mathbb{R}$ by

$$
\lambda(x, r)=\mu(x, N(x, r)) \quad \text { a.e. } x \in \Omega, \forall r \in \mathbb{R} .
$$

Then $\lambda$ is a Carathéodory function which satisfies (2.1) (for some new modulus of continuity) and (2.4), i.e.

$$
c_{1} \leq \lambda(x, r) \leq c_{2} \quad \text { a.e. } x \in \Omega, \forall r \in \mathbb{R}
$$

and which is nonincreasing in $r$ when $\mu$ satisfies (2.7), i.e.

$$
\frac{\partial \lambda}{\partial r}(x, r) \leq 0 \quad \text { in } \mathscr{D}^{\prime}(\Omega \times \mathbb{R}) ;
$$

(note that this is the only place where hypothesis (2.7) is used in the present Subsection.)

Then we have

$$
\begin{gathered}
\mu(x, \theta(t, x))=\lambda(x, \tau(t, x)) \\
\sigma(t, x)=\mu(x, \theta(t, x)) \frac{\partial v}{\partial x}(t, x)=\lambda(x, \tau(t, x))\left(\frac{\partial u}{\partial x}(t, x)+\frac{\partial \bar{v}}{\partial x}(t, x)\right) .
\end{gathered}
$$

It is now straightforward to prove the following equivalence result.

Theorem 2.2 (Equivalence) Assume that hypotheses (2.1)-(2.6) and (2.8)-(2.12) hold true, and define $\bar{v}, u_{0}, M, N$ and $\lambda$ by (2.43), (2.44), (2.52), (2.53) and (2.59). Then $(v, \theta)$ is a solution of (2.13)-(2.19) if and only if $u$ and $\tau$ defined by (2.45) and (2.58), namely

$$
u(t, x)=v(t, x)-\bar{v}(t, x), \quad \tau(t, x)=M(x, \theta(t, x)),
$$

satisfy

$$
\begin{gathered}
u \in L^{\infty}\left(0, T ; H_{0}^{1}(\Omega)\right) \cap H^{1}\left(0, T ; L^{2}(\Omega)\right) \\
\tau \in W^{1,1}\left(0, T ; L^{1}(\Omega)\right) \\
\rho(x) \frac{\partial u}{\partial t}-\frac{\partial}{\partial x}\left(\lambda(x, \tau)\left(\frac{\partial u}{\partial x}+\frac{\partial \bar{v}}{\partial x}\right)\right)=f-\rho(x) \frac{\partial \bar{v}}{\partial t} \quad \text { in } \mathscr{D}^{\prime}(Q), \\
\frac{\partial \tau}{\partial t}=\left|\lambda(x, \tau)\left(\frac{\partial u}{\partial x}+\frac{\partial \bar{v}}{\partial x}\right)\right|^{2} \quad \text { in } \mathscr{D}^{\prime}(Q), \\
u(0, x)=u_{0}(x) \quad \text { a.e. } x \in \Omega \\
\tau(0, x)=0 \quad \text { a.e. } x \in \Omega .
\end{gathered}
$$


Moreover the stress $\sigma$ defined by (2.20), namely

$$
\sigma=\mu(x, \theta) \frac{\partial v}{\partial x}=\lambda(x, \tau)\left(\frac{\partial u}{\partial x}+\frac{\partial \bar{v}}{\partial x}\right)
$$

satisfies

$$
\sigma \in L^{\infty}\left(0, T ; L^{2}(\Omega)\right) \cap L^{2}\left(0, T ; H^{1}(\Omega)\right) .
$$

Finally the norms of $v$ and $\theta$ in the spaces $L^{\infty}\left(0, T ; H^{1}(\Omega)\right) \cap$ $\cap H^{1}\left(0, T ; L^{2}(\Omega)\right)$ and $W^{1,1}\left(0, T ; L^{1}(\Omega)\right)$ and the norms of $u$ and $\tau$ in the spaces $L^{\infty}\left(0, T ; H_{0}^{1}(\Omega)\right) \cap H^{1}\left(0, T ; L^{2}(\Omega)\right)$ and $W^{1,1}\left(0, T ; L^{1}(\Omega)\right)$ are equivalent, with constants in the equivalences which depend only on $c_{1}, c_{2}, c_{3}, c_{4},(b-a)$ and $J$, when the data satisfy

$$
\left\|v_{a}\right\|_{H^{1}(0, T)}+\left\|v_{b}\right\|_{H^{1}(0, T)}+\left\|\theta_{0}\right\|_{L^{1}(\Omega)} \leq J .
$$

Note that the initial conditions (2.65) and (2.66) have a meaning since $H^{1}\left(0, T ; L^{2}(\Omega)\right) \subset C^{0}\left([0, T] ; L^{2}(\Omega)\right) \quad$ and $\quad$ since $\quad W^{1,1}\left(0, T ; L^{1}(\Omega)\right) \subset$ $\subset C^{0}\left([0, T] ; L^{1}(\Omega)\right)$.

Theorem 2.1 then results from the equivalence Theorem 2.2 and from the following existence Theorem, that we will prove in Subsection 2.4.

Theorem 2.3 (Existence for the transformed system) Assume that the hypotheses of Theorem 2.1 hold true, and define $\bar{v}, u_{0}, M, N$ and $\lambda$ by (2.43), (2.44), (2.52), (2.53) and (2.59). Then there exists at least one couple $(u, \tau)$ which satisfy (2.61)-(2.66).

Moreover the stress $\sigma$ defined by (2.67) satisfies (2.68).

Finally for this solution, the following a priori estimates hold true

$$
\begin{gathered}
\|u\|_{L^{\infty}\left(0, T ; H_{0}^{1}(\Omega)\right)}+\left\|\frac{\partial u}{\partial t}\right\|_{L^{2}\left(0, T ; L^{2}(\Omega)\right)} \leq D^{\star}, \\
\|\tau\|_{L^{\infty}\left(0, T ; L^{1}(\Omega)\right)}+\left\|\frac{\partial \tau}{\partial t}\right\|_{L^{1}\left(0, T ; L^{1}(\Omega)\right)} \leq D^{\star}, \\
\|\sigma\|_{L^{\infty}\left(0, T ; L^{2}(\Omega)\right)}+\|\sigma\|_{L^{2}\left(0, T ; H^{1}(\Omega)\right)} \leq D^{\star},
\end{gathered}
$$

where $D^{\star}$ denotes a constant which depends only on $c_{1}, c_{2}, c_{5}, c_{6},(b-a)$ and $L$, when the data satisfy

$$
\|f\|_{L^{2}\left(0, T ; L^{2}(\Omega)\right)}+\left\|v_{a}\right\|_{H^{1}(0, T)}+\left\|v_{b}\right\|_{H^{1}(0, T)}+\left\|u_{0}\right\|_{H_{0}^{1}(\Omega)} \leq L .
$$

2.3 Definition of the approximating problem

The proof of Theorem 2.3 that we will present is based on Galerkin's approximation by $\mathbb{P}^{1}$ (in $u$ ) and $\mathbb{P}^{0}$ (in $\tau$ ) finite elements.

We divide $\Omega=(a, b)$ into $n$ intervals $I_{n}^{k}$ of length $\frac{b-a}{n}$ defined by

$$
I_{n}^{k}=\left[a+\frac{k}{n}(b-a), a+\frac{(k+1)}{n}(b-a)\right], \quad k=0,1, \ldots, n-1,
$$


whose characteristic functions $\chi_{n}^{k}$ are given by

$$
\chi_{n}^{k}(x)= \begin{cases}1 \quad \text { if } x \in\left[a+\frac{k}{n}(b-a), a+\frac{(k+1)}{n}(b-a)\right], \\ 0 \quad \text { otherwise, } \quad k=0,1, \ldots, n-1,\end{cases}
$$

and we denote by $w_{n}^{j}$ the "hat" functions which are the elements of the $\mathbb{P}^{1}$ basis, i.e.

$$
w_{n}^{j}(x)=\left\{\begin{array}{r}
1-\frac{n}{b-a} \mid x-\left(a+\frac{j}{n}(b-a) \mid\right. \\
\quad \text { if } x \in\left[a+\frac{(j-1)}{n}(b-a), a+\frac{(j+1)}{n}(b-a)\right], \\
0 \quad \text { otherwise, } \quad j=1, \ldots, n-1 .
\end{array}\right.
$$

\subsubsection{Definition of $\lambda_{n}$ and $U_{n}$}

Let us define an approximation $\lambda_{n}(x, r)$ of the function $\lambda(x, r)$ and an approximation $U_{n}(x)$ of the initial condition $u_{0}(x)$.

Since $\lambda(x, r)=\mu(x, N(x, r))$, and since $\mu$ is continuous and non increasing in $s$ while $N$ is continuous and strictly increasing in $r$, the function $\lambda(x, r)$ is continuous and non increasing in $s$. We first define a regularization $\tilde{\lambda}_{n}(x, r)$ which is $C^{1}$ and non increasing in $r$ for each fixed $n$, which satisfies the bound

$$
c_{1} \leq \tilde{\lambda}_{n}(x, r) \leq c_{2} \quad \text { a.e. } x \in \Omega, \forall r \in \mathbb{R},
$$

and which is such that

$$
\left\{\begin{array}{l}
x \rightarrow \tilde{\lambda}_{n}(x, r) \quad \text { is measurable } \forall r \in \mathbb{R}, \\
\left|\tilde{\lambda}_{n}(x, r)-\tilde{\lambda}_{n}\left(x, r^{\prime}\right)\right| \leq \tilde{\omega}\left(r,\left|r-r^{\prime}\right|\right), \quad \text { a.e. } x \in \mathbb{R}, \forall r, r^{\prime} \in \mathbb{R}, \\
\text { where } \tilde{\omega}(r, \hat{r}) \rightarrow 0 \quad \text { when } \hat{r} \rightarrow 0,
\end{array}\right.
$$

such a regularization can be obtained e.g. by convolution of $\lambda(x, r)$ with respect to $r$; it is superfluous when $\mu$ (and thus $\lambda$ ) is $C^{1}$ in $s$; this regularization will make licit the derivation of the first a priori estimate below.

We then define $\lambda_{n}(x, r)$ as the mean value (in $\left.x\right)$ of $\tilde{\lambda}_{n}(x, r)$ over the intervals $I_{n}^{k}$ of length $\frac{b-a}{n}$, i.e.

$$
\lambda_{n}(x, r)=\sum_{k=0}^{n-1} \chi_{n}^{k}(x)\left[\frac{n}{b-a} \int_{\Omega} \chi_{n}^{k}\left(x^{\prime}\right) \tilde{\lambda}_{n}\left(x^{\prime}, r\right) d x^{\prime}\right] .
$$


It is easy to prove that the function $\lambda_{n}: \Omega \times \mathbb{R} \rightarrow \mathbb{R}$ satisfies for each $n$ the following properties

$\lambda_{n}$ is constant in $x$ on each interval $I_{n}^{k}$, and is $C^{1}$ and non increasing in $r$,

$$
\begin{gathered}
c_{1} \leq \lambda_{n}(x, r) \leq c_{2} \quad \text { a.e. } x \in \Omega, \forall r \in \mathbb{R}, \\
\text { as } n \rightarrow \infty, \quad \lambda_{n}\left(x, r_{n}\right) \rightarrow \lambda(x, r) \quad \text { if } r_{n} \rightarrow r, \quad \text { a.e. } x \in \Omega .
\end{gathered}
$$

On the other hand we define $U_{n}$ as the projection in $H_{0}^{1}(\Omega)$ of $u_{0}$ on the space generated by the functions $w_{n}^{j}, j=1, \ldots, n-1$, i.e.

$$
U_{n}(x)=\sum_{j=1}^{n-1} U_{n}^{j} w_{n}^{j}
$$

where the coefficients $U_{n}^{j} \in \mathbb{R}$ are defined by the system of equations

$$
\sum_{j=1}^{n-1} U_{n}^{j}<w_{n}^{j}, w_{n}^{i}>=<u_{0}, w_{n}^{i}>, \quad i=1, \ldots, n-1,
$$

in which $<,>$ denotes the scalar product of $H_{0}^{1}(\Omega)$, i.e.

$$
<\varphi, \psi>=\int_{\Omega} \frac{d \varphi}{d x} \frac{d \psi}{d x} d x \quad \forall \varphi, \psi \in H_{0}^{1}(\Omega) .
$$

Therefore

$$
\begin{gathered}
\left\|U_{n}\right\|_{H_{0}^{1}(\Omega)} \leq C\left\|u_{0}\right\|_{H_{0}^{1}(\Omega)}, \\
U_{n} \rightarrow u_{0} \quad \text { in } H_{0}^{1}(\Omega) \text { strong. }
\end{gathered}
$$

\subsubsection{Approximating problem}

We now define the approximating problem in the following way. We look for functions $\left(u_{n}, \tau_{n}\right)$ (or more exactly for their coefficients $u_{n}^{j}(t) \in H^{1}(0, T), j=$ $=1, \ldots, n-1$, and $\left.\tau_{n}^{k}(t) \in W^{1,1}(0, T), k=0,1, \ldots, n-1\right)$, which are solutions of the approximating system

$$
\begin{gathered}
u_{n}(t, x)=\sum_{j=1}^{n-1} u_{n}^{j}(t) w_{n}^{j}(x), \\
\tau_{n}(t, x)=\sum_{k=0}^{n-1} \tau_{n}^{k}(t) \chi_{n}^{k}(x), \\
\left\{\begin{array}{l}
\int_{\Omega} \rho(x) \frac{\partial u_{n}}{\partial t} w_{n}^{i} d x+\int_{\Omega} \lambda_{n}\left(x, \tau_{n}\right)\left(\frac{\partial u_{n}}{\partial x}+\frac{\partial \bar{v}}{\partial x}\right) \frac{\partial w_{n}^{i}}{\partial x} d x= \\
=\int_{\Omega} f w_{n}^{i} d x-\int_{\Omega} \rho(x) \frac{\partial \bar{v}}{\partial t} w_{n}^{i} d x, \quad i=1, \ldots, n-1, \quad t \in(0, T),
\end{array}\right.
\end{gathered}
$$




$$
\begin{gathered}
\frac{\partial \tau_{n}}{\partial t}=\left|\lambda_{n}\left(x, \tau_{n}\right)\left(\frac{\partial u_{n}}{\partial x}+\frac{\partial \bar{v}}{\partial x}\right)\right|^{2}, \quad(t, x) \in(0, T) \times \Omega, \\
u_{n}(0, x)=U_{n}(x), \quad x \in \Omega, \\
\tau_{n}(0, x)=0, \quad x \in \Omega,
\end{gathered}
$$

where $\lambda_{n}$ and $U_{n}$ are defined by (2.74) and (2.78). Observe that since $\tau_{n}(t)$, $\lambda_{n}(x, r), \frac{\partial u_{n}}{\partial x}$ and $\frac{\partial \bar{v}}{\partial x}$ are constant (in $x$ ) on each interval $I_{n}^{k}$, equation (2.84) is equivalent to $n$ ordinary differential equations or to

$$
\begin{gathered}
\int_{\Omega} \frac{\partial \tau_{n}}{\partial t} \chi_{n}^{k} d x=\int_{\Omega}\left|\lambda_{n}\left(x, \tau_{n}\right)\left(\frac{\partial u_{n}}{\partial x}+\frac{\partial \bar{v}_{n}}{\partial x}\right)\right|^{2} \chi_{n}^{k} d x \\
k=0,1, \ldots, n-1, \quad t \in(0, T)
\end{gathered}
$$

\subsubsection{Local existence of the approximating solution}

The approximating system (2.81)-(2.86) is nothing but the ordinary differential equation

$$
\left\{\begin{array}{l}
\frac{d Z(t)}{d t}=F_{n}(t, Z(t)), \quad t \in(0, T), \\
Z(0)=Z_{0},
\end{array}\right.
$$

where $Z:[0, T] \rightarrow \mathbb{R}^{n-1+n}$ is defined by

$$
Z(t)=\left(u_{n}^{1}(t), \ldots, u_{n}^{n-1}(t), \tau_{n}^{0}(t), \ldots, \tau_{n}^{n-1}(t)\right),
$$

and where $F_{n}(t, z)$ is $C^{1}$ in $z$ and $L^{2}$ in $t$. Thus there exists a solution $Z$ of (2.87) (and therefore of the approximating system) which belongs to $H^{1}\left(0, T^{\star} ; \mathbb{R}^{n-1+n}\right)$ on the interval $\left(0, T^{\star}\right)$ for some $T^{\star}$ with $0<T^{\star} \leq T$. The a priori estimates that we will obtain in the next Subsection will imply that actually $T^{\star}=T$.

2.4 Convergence of the approximating solution

In this Subsection we prove that the approximating solution $\left(u_{n}, \tau_{n}\right)$ of $(2.81)-$ (2.86) converges to a solution $(u, \tau)$ of (2.61)-(2.66) (see Theorem 2.4 below).

This will prove the existence Theorem 2.3 for the transformed system.

Theorem 2.4 (Convergence of Galerkin's approximation) The solution $\left(u_{n}, \tau_{n}\right)$ of the approximating system (2.81)-(2.86) exists on $(0, T)$ and a subsequence, still denoted by the index $n$, satisfies

$$
\begin{gathered}
u_{n} \rightarrow u \quad \text { in } L^{\infty}\left(0, T ; H_{0}^{1}(\Omega)\right) \text { weak } \star \\
\frac{\partial u_{n}}{\partial x} \rightarrow \frac{\partial u}{\partial x} \quad \text { in } L^{2}\left(0, T ; L^{2}(\Omega)\right) \text { strong }
\end{gathered}
$$




$$
\begin{gathered}
\frac{\partial u_{n}}{\partial t} \rightarrow \frac{\partial u}{\partial t} \quad \text { in } L^{2}\left(0, T ; L^{2}(\Omega)\right) \text { weak } \\
\tau_{n} \rightarrow \tau \quad \text { in } W^{1,1}\left(0, T ; L^{1}(\Omega)\right) \text { strong }
\end{gathered}
$$

where $(u, \tau)$ is a solution of (2.61)-(2.66).

Proof The proof of this Theorem will be done in five steps.

\section{First step: a priori estimates}

Multiplication of (2.83) by $\frac{d u_{n}^{i}}{d t}(t)$ and addition from $i=1$ to $i=n-1$ yields

$$
\left\{\begin{array}{l}
\int_{\Omega} \rho(x)\left|\frac{\partial u_{n}}{\partial t}\right|^{2}+\int_{\Omega} \lambda_{n}\left(x, \tau_{n}\right)\left(\frac{\partial u_{n}}{\partial x}+\frac{\partial \bar{v}}{\partial x}\right)\left(\frac{\partial^{2} u_{n}}{\partial x \partial t}+\frac{\partial^{2} \bar{v}}{\partial x \partial t}\right) d x= \\
=\int_{\Omega} f \frac{\partial u_{n}}{\partial t} d x-\int_{\Omega} \rho(x) \frac{\partial \bar{v}}{\partial t} \frac{\partial u_{n}}{\partial t} d x+ \\
+\int_{\Omega} \lambda_{n}\left(x, \tau_{n}\right)\left(\frac{\partial u_{n}}{\partial x}+\frac{\partial \bar{v}}{\partial x}\right) \frac{\partial^{2} \bar{v}}{\partial x \partial t} d x .
\end{array}\right.
$$

For what concerns the second term of (2.92), using the fact that $\lambda_{n}$ is $C^{1}$ in $r$, the fact that $\frac{\partial}{\partial t}\left(\lambda_{n}\left(x, \tau_{n}\right)\right)=\frac{\partial \lambda_{n}}{\partial r}\left(x, \tau_{n}\right) \frac{\partial \tau_{n}}{\partial t}$ and equation (2.84), we have

$$
\begin{aligned}
& \frac{d}{d t}\left[\frac{1}{2} \int_{\Omega} \lambda_{n}\left(x, \tau_{n}\right)\left|\frac{\partial u_{n}}{\partial x}+\frac{\partial \bar{v}}{\partial x}\right|^{2} d x\right]= \\
& =\int_{\Omega} \lambda_{n}\left(x, \tau_{n}\right)\left(\frac{\partial u_{n}}{\partial x}+\frac{\partial \bar{v}}{\partial x}\right)\left(\frac{\partial^{2} u_{n}}{\partial x \partial t}+\frac{\partial^{2} \bar{v}}{\partial x \partial t}\right) d x+ \\
& +\frac{1}{2} \int_{\Omega} \frac{\partial \lambda_{n}}{\partial r}\left(x, \tau_{n}\right)\left|\lambda_{n}\left(x, \tau_{n}\right)\right|^{2}\left|\frac{\partial u_{n}}{\partial x}+\frac{\partial \bar{v}}{\partial x}\right|^{4} d x
\end{aligned}
$$

Since $\frac{\partial \lambda_{n}}{\partial r}(x, r) \leq 0$, we have

$$
\begin{aligned}
& \frac{d}{d t}\left[\frac{1}{2} \int_{\Omega} \lambda_{n}\left(x, \tau_{n}\right)\left|\frac{\partial u_{n}}{\partial x}+\frac{\partial \bar{v}}{\partial x}\right|^{2} d x\right] \leq \\
& \leq \int_{\Omega} \lambda_{n}\left(x, \tau_{n}\right)\left(\frac{\partial u_{n}}{\partial x}+\frac{\partial \bar{v}}{\partial x}\right)\left(\frac{\partial^{2} u_{n}}{\partial x \partial t}+\frac{\partial^{2} \bar{v}}{\partial x \partial t}\right) d x
\end{aligned}
$$


Therefore integrating (2.92) in time from 0 to $t$ we obtain

$$
\begin{aligned}
& \int_{0}^{t} \int_{\Omega} \rho(x)\left|\frac{\partial u_{n}}{\partial t}\right|^{2} d x d t^{\prime}+\frac{1}{2} \int_{\Omega} \lambda_{n}\left(x, \tau_{n}(t, x)\right)\left|\frac{\partial u_{n}}{\partial x}(t, x)+\frac{\partial \bar{v}}{\partial x}(t, x)\right|^{2} d x \leq \\
& \leq \frac{1}{2} \int_{\Omega} \lambda_{n}(x, 0)\left|\frac{\partial U_{n}}{\partial x}+\frac{\partial \bar{v}}{\partial x}(0, x)\right|^{2} d x+ \\
& +\int_{0}^{t} \int_{\Omega} f \frac{\partial u_{n}}{\partial t} d x d t^{\prime}-\int_{0}^{t} \int_{\Omega} \rho(x) \frac{\partial \bar{v}}{\partial t} \frac{\partial u_{n}}{\partial t} d x d t^{\prime}+ \\
& +\int_{0}^{t} \int_{\Omega} \lambda_{n}\left(x, \tau_{n}\right)\left(\frac{\partial u_{n}}{\partial x}+\frac{\partial \bar{v}}{\partial x}\right) \frac{\partial^{2} \bar{v}}{\partial x \partial t} d x d t^{\prime} .
\end{aligned}
$$

Using the bounds (2.6) and (2.76) on $\rho$ and $\lambda_{n}$ as well as Young's inequality we get

$$
\begin{aligned}
& c_{5} \int_{0}^{t} \int_{\Omega}\left|\frac{\partial u_{n}}{\partial t}\right|^{2} d x d t^{\prime}+\frac{1}{2} c_{1} \int_{\Omega}\left|\frac{\partial u_{n}}{\partial x}(t, x)+\frac{\partial \bar{v}}{\partial x}(t, x)\right|^{2} d x \leq \\
& \leq \frac{1}{2} c_{2} \int_{\Omega}\left|\frac{\partial U_{n}}{\partial x}+\frac{\partial \bar{v}}{\partial x}(0, x)\right|^{2} d x+\frac{c_{5}}{4} \int_{0}^{t} \int_{\Omega}\left|\frac{\partial u_{n}}{\partial t}\right|^{2} d x d t^{\prime}+\frac{1}{c_{5}} \int_{0}^{t} \int_{\Omega}|f|^{2} d x d t^{\prime}+ \\
& +\frac{c_{5}}{4} \int_{0}^{t} \int_{\Omega}\left|\frac{\partial u_{n}}{\partial t}\right|^{2} d x d t^{\prime}+\frac{c_{6}^{2}}{c_{5}} \int_{0}^{t} \int_{\Omega}\left|\frac{\partial \bar{v}}{\partial t}\right|^{2} d x d t^{\prime}+ \\
& +\frac{c_{1}}{4} \int_{0}^{t} \int_{\Omega}\left|\frac{\partial u_{n}}{\partial x}+\frac{\partial \bar{v}}{\partial x}\right|^{2} d x d t^{\prime}+\frac{c_{2}^{2}}{c_{1}} \int_{0}^{t} \int_{\Omega}\left|\frac{\partial^{2} \bar{v}}{\partial x \partial t}\right|^{2} d x d t^{\prime} .
\end{aligned}
$$

From Gronwall's inequality, from the definition (2.43) of $\bar{v}$ and from the estimate (2.79) on $U_{n}$, we obtain

$$
\int_{0}^{T^{\star}} \int_{\Omega}\left|\frac{\partial u_{n}}{\partial t}\right|^{2} d x d t^{\prime}+\sup _{0<t<T^{\star}} \int_{\Omega}\left|\frac{\partial u_{n}}{\partial x}(t, x)+\frac{\partial \bar{v}}{\partial x}(t, x)\right|^{2} d x \leq D^{\star},
$$

where the constant $D^{\star}$ depends only on $c_{1}, c_{2}, c_{5}, c_{6},\|f\|_{L^{2}\left(0, T ; L^{2}(\Omega)\right)},\left\|v_{a}\right\|_{H^{1}(0, T)}$, $\left\|v_{b}\right\|_{H^{1}(0, T)}$ and $\left\|u_{0}\right\|_{H_{0}^{1}(\Omega)}$.

From (2.84) and (2.86), we immediately deduce from (2.93) that

$$
\left\|\frac{\partial \tau_{n}}{\partial t}\right\|_{L^{\infty}\left(0, T^{\star} ; L^{1}(\Omega)\right)} \leq D^{\star}
$$

where $D^{\star}$ is a constant, possibly different of the constant which appears in (2.93), but which depends only on the same quantities.

For $n$ fixed, the estimates (2.93) and (2.94) provide in particular an a priori estimate (independent of $\left.T^{\star}\right)$ in $L^{\infty}\left(0, T^{\star} ; \mathbb{R}^{n-1+n}\right)$ for the solution $Z(t)$ of the ordinary differential equation (2.87). This implies the global existence of $Z$ on $(0, T)$, i.e. $T^{\star}=T$. 
In view of (2.93) and (2.94), we can extract a subsequence, still denoted by the index $n$, such that

$$
\begin{aligned}
& u_{n} \rightarrow u \quad \text { in } L^{\infty}\left(0, T ; H_{0}^{1}(\Omega)\right) \text { weak } \star \\
& \frac{\partial u_{n}}{\partial t} \rightarrow \frac{\partial u}{\partial t} \quad \text { in } L^{2}\left(0, T ; L^{2}(\Omega)\right) \text { weak }
\end{aligned}
$$

for some $u$ which satisfies

$$
u \in L^{\infty}\left(0, T ; H_{0}^{1}(\Omega)\right), \quad \frac{\partial u}{\partial t} \in L^{2}\left(0, T ; L^{2}(\Omega)\right),
$$

and which thus meets the regularity requirement (2.61). Convergences (2.88) and (2.90) of Theorem 2.4 (which are nothing but convergences (2.95) and (2.96)) are also proved, and estimate (2.70) follows from (2.93) and from weak lower semicontinuity.

Since (2.95) and (2.96) imply that

$$
u_{n} \rightarrow u \quad \text { in } C^{0}\left([0, T] ; L^{2}(\Omega)\right) \text { strong, }
$$

passing to the limit in the initial condition (2.85) with the help of (2.80) yields

$$
u(0, x)=u_{0}(x) \quad \text { a.e. } x \in \Omega .
$$

Therefore $u$ satisfies the initial condition (2.65).

\section{Second step: study of the approximating stress}

We define $\sigma_{n}$, the natural approximation of the stress, by

$$
\sigma_{n}(t, x)=\lambda_{n}\left(x, \tau_{n}(t, x)\right)\left(\frac{\partial u_{n}}{\partial x}(t, x)+\frac{\partial \bar{v}}{\partial x}(t, x)\right) .
$$

In view of the bound (2.76) on $\lambda_{n}$ and of the estimate (2.93) on $u_{n}, \sigma_{n}$ satisfies

$$
\left\|\sigma_{n}\right\|_{L^{\infty}\left(0, T ; L^{2}(\Omega)\right)} \leq D^{\star},
$$

where $D^{\star}$ is a constant possibly different of the constants which appear in (2.93) and in (2.94), but which depends only on the same quantities. We can thus extract a new subsequence, still denoted by the index $n$, such that

$$
\sigma_{n} \rightarrow \sigma \quad \text { in } L^{\infty}\left(0, T ; L^{2}(\Omega)\right) \text { weak } \star,
$$

for some $\sigma \in L^{\infty}\left(0, T ; L^{2}(\Omega)\right)$.

We also define a function $s_{n}$ by

$$
\begin{gathered}
\frac{\partial s_{n}}{\partial x}=\rho(x) \frac{\partial u_{n}}{\partial t}-f+\rho(x) \frac{\partial \bar{v}}{\partial t} \quad \text { in } \mathscr{D}^{\prime}(Q), \\
\int_{\Omega} s_{n}(t, x) d x=\int_{\Omega} \sigma_{n}(t, x) d x .
\end{gathered}
$$


Since the right hand side of (2.102) belongs to $L^{2}\left(0, T ; L^{2}(\Omega)\right)$, conditions (2.102) and (2.103) define a function $s_{n}$ in $L^{2}\left(0, T ; H^{1}(\Omega)\right)$. We then define $\bar{s}_{n}$ as the mean value (in $x$ ) of $s_{n}$ on the intervals $I_{n}^{k}$ of length $\frac{b-a}{n}$, i.e.

$$
\bar{s}_{n}(t, x)=\sum_{k=0}^{n-1} \chi_{n}^{k}(x)\left[\frac{n}{b-a} \int_{\Omega} \chi_{n}^{k}\left(x^{\prime}\right) s_{n}\left(x^{\prime}, t\right) d x^{\prime}\right] .
$$

Actually we have the following surprising result

$$
\sigma_{n}=\bar{s}_{n},
$$

that we will now prove.

Using the approximating equation (2.83), the definition (2.99) of $\sigma_{n}$ and then the definition (2.102) of $\frac{\partial s_{n}}{\partial x}$, we have, since $w_{n}^{j} \in H_{0}^{1}(\Omega)$,

$$
\begin{aligned}
\int_{\Omega} \sigma_{n} \frac{\partial w_{n}^{i}}{\partial x} d x & =-\int_{\Omega} \rho(x) \frac{\partial u_{n}}{\partial t} w_{n}^{i} d x+\int_{\Omega} f w_{n}^{i} d x-\int_{\Omega} \rho(x) \frac{\partial \bar{v}}{\partial t} w_{n}^{i} d x= \\
& =-\int_{\Omega} \frac{\partial s_{n}}{\partial x} w_{n}^{i} d x=\int_{\Omega} s_{n} \frac{\partial w_{n}^{i}}{\partial x} d x, \quad i=1, \ldots, n-1 .
\end{aligned}
$$

Since $\frac{\partial w_{n}^{i}}{\partial x}$ is constant on each interval $I_{n}^{k}$, we have

$$
\int_{\Omega} s_{n} \frac{\partial w_{n}^{i}}{\partial x} d x=\int_{\Omega} \bar{s}_{n} \frac{\partial w_{n}^{i}}{\partial x} d x, \quad i=1, \ldots, n-1,
$$

and thus

$$
\int_{\Omega}\left(\sigma_{n}-\bar{s}_{n}\right) \frac{\partial w_{n}^{i}}{\partial x} d x=0, \quad i=1, \ldots, n-1 .
$$

But by their very definitions, $\sigma_{n}$ and $\bar{s}_{n}$ are constant (in $x$ ) on each interval $I_{n}^{k}$, and therefore there exist functions $h_{n}^{k}(t), k=0,1, \ldots, n-1$, such that

$$
\sigma_{n}-\bar{s}_{n}=\sum_{k=0}^{n-1} h_{n}^{k}(t) \chi_{n}^{k}(x) .
$$

Then (2.106) and the explicit form of $\frac{\partial w_{n}^{i}}{\partial x}$, i.e.

$$
\frac{\partial w_{n}^{i}}{\partial x}(x)=\frac{n}{b-a}\left(\chi_{n}^{i-1}(x)-\chi_{n}^{i}(x)\right), \quad i=1, \ldots, n-1,
$$

imply that $h_{n}^{k}(t)$ does not depend on $k$, i.e.

$$
h_{n}^{k}(t)=h_{n}(t), \quad k=0, \ldots, n-1,
$$

for some function $h_{n}(t)$, from which we deduce that

$$
\sigma_{n}-\bar{s}_{n}=h_{n}(t) \quad \text { on } Q,
$$


and by (2.103) that

$$
(b-a) h_{n}(t)=\int_{\Omega}\left(\sigma_{n}-\bar{s}_{n}\right) d x=0,
$$

i.e. that $\sigma_{n}=\bar{s}_{n}$ as required.

Since $\frac{\partial u_{n}}{\partial t}$ is bounded in $L^{2}\left(0, T ; L^{2}(\Omega)\right)$ (see (2.93)), and since $\int_{\Omega} \sigma_{n}(t, x) d x$ is bounded in $L^{\infty}(0, T)$ because $\sigma_{n}$ is bounded in $L^{\infty}\left(0, T ; L^{2}(\Omega)\right)$ (see (2.100)), we deduce from the definition (2.102)-(2.103) of $s_{n}$ that

$$
\left\|s_{n}\right\|_{L^{2}\left(0, T ; H^{1}(\Omega)\right)} \leq D^{\star} .
$$

We now claim that

$$
\left\|s_{n}-\bar{s}_{n}\right\|_{L^{2}\left(0, T ; L^{2}(\Omega)\right)}^{2} \leq\left(\frac{b-a}{n}\right)^{2}\left\|s_{n}\right\|_{L^{2}\left(0, T ; H^{1}(\Omega)\right)}^{2} .
$$

Indeed, for every function $\psi \in H^{1}(\Omega)$, denoting by $\bar{\psi}_{n}$ the mean value of $\psi$ on the intervals $I_{n}^{k}$ of lenght $\frac{b-a}{n}$, i.e.

$$
\bar{\psi}_{n}(x)=\sum_{k=0}^{n-1} \chi_{n}^{k}(x)\left[\frac{n}{b-a} \int_{\Omega} \chi_{n}^{k}\left(x^{\prime}\right) \psi\left(x^{\prime}\right) d x^{\prime}\right]
$$

one has

$$
\left\|\psi-\bar{\psi}_{n}\right\|_{L^{2}(\Omega)}^{2} \leq\left(\frac{b-a}{n}\right)^{2}\|\psi\|_{H^{1}(\Omega)}^{2} ;
$$

this assertion follows by addition from $k=0$ to $k=n-1$ of the following inequality, in which ones takes $\alpha=a+\frac{k}{n}(b-a)$ and $\beta=a+\frac{(k+1)}{n}(b-a)$

$$
\left\|\psi-\frac{1}{\beta-\alpha} \int_{\alpha}^{\beta} \psi\left(x^{\prime}\right) d x^{\prime}\right\|_{L^{2}(\alpha, \beta)}^{2} \leq(\beta-\alpha)^{2}\left\|\frac{\partial \psi}{\partial x}\right\|_{L^{2}(\alpha, \beta)}^{2} ;
$$

the latest inequality is nothing but the one dimensional Poincaré-Wirtinger inequality and can be proved in an elementary way.

In conclusion, we deduce from (2.105), (2.108) and (2.107) that

$$
\left\|\sigma_{n}-s_{n}\right\|_{L^{2}\left(0, T ; L^{2}(\Omega)\right)}^{2}=\left\|\bar{s}_{n}-s_{n}\right\|_{L^{2}\left(0, T ; L^{2}(\Omega)\right)}^{2} \leq\left(\frac{b-a}{n}\right)^{2} D^{\star},
$$

which combined with (2.101) implies that $s_{n}$ tends to $\sigma$ in $L^{2}\left(0, T ; L^{2}(\Omega)\right)$ weak. Then (2.107) implies that $\sigma \in L^{2}\left(0, T ; H^{1}(\Omega)\right)$.

This result and (2.101) prove the regularity (2.68) on $\sigma$, while (2.100), (2.101) and (2.107) prove the a priori estimate (2.72). 


\section{Third step: strong convergence of $\tau_{n}$ in $L^{1}(Q)$}

Using the definition (2.99) of $\sigma_{n}$ in (2.84) and recalling (2.86), we have

$$
\frac{\partial \tau_{n}}{\partial t}=\left|\sigma_{n}\right|^{2} \quad \text { in } \mathscr{D}^{\prime}(Q), \quad \tau_{n}(0, x)=0,
$$

which we rewrite as

$$
\left\{\begin{array}{l}
\tau_{n}=\zeta_{n}+\hat{\zeta}_{n}, \\
\frac{\partial \zeta_{n}}{\partial t}=\left|s_{n}\right|^{2}, \quad \zeta_{n}(0, x)=0, \\
\frac{\partial \hat{\zeta}_{n}}{\partial t}=\left|\sigma_{n}\right|^{2}-\left|s_{n}\right|^{2}, \quad \hat{\zeta}_{n}(0, x)=0 .
\end{array}\right.
$$

From (2.100) and (2.109) we deduce that

$$
\left|\sigma_{n}\right|^{2}-\left|s_{n}\right|^{2}=2 \sigma_{n}\left(\sigma_{n}-s_{n}\right)-\left(\sigma_{n}-s_{n}\right)^{2} \rightarrow 0 \text { in } L^{1}\left(0, T ; L^{1}(\Omega)\right) \text { strong, }
$$

and thus that $\hat{\zeta}_{n}(t, x)=\int_{0}^{t} \frac{\partial \hat{\zeta}_{n}}{\partial t}\left(t^{\prime}, x\right) d t^{\prime}$ satisfies

$$
\left\|\hat{\zeta}_{n}\right\|_{L^{\infty}\left(0, T ; L^{1}(\Omega)\right)} \rightarrow 0
$$

Let us now study $\zeta_{n}$. Since $s_{n}$ is bounded in $L^{2}\left(0, T ; H^{1}(\Omega)\right)$ (see (2.107)), $\frac{\partial \zeta_{n}}{\partial t}$ is bounded in $L^{1}\left(0, T ; L^{1}(\Omega)\right.$, and $\zeta_{n}(t, x)=\int_{0}^{t} \frac{\partial \zeta_{n}}{\partial t}\left(t^{\prime}, x\right) d t^{\prime}$ is bounded in $L^{\infty}\left(0, T ; L^{1}(\Omega)\right)$. On the other hand, $2 s_{n} \frac{\partial s_{n}}{\partial x}$ is bounded in $L^{1}\left(0, T ; L^{1}(\Omega)\right)$, still because of (2.107). Since

$$
\frac{\partial}{\partial t}\left(\frac{\partial \zeta_{n}}{\partial x}\right)=2 s_{n} \frac{\partial s_{n}}{\partial x}, \quad \frac{\partial \zeta_{n}}{\partial x}(0, x)=0
$$

we obtain that $\frac{\partial \zeta_{n}}{\partial x}$ is bounded in $L^{\infty}\left(0, T ; L^{1}(\Omega)\right)$. Therefore $\zeta_{n}$ is bounded in $W^{1,1}(Q)$, and extracting if necessary a new subsequence, still denoted by the index $n$, we have

$$
\zeta_{n} \rightarrow \tau \text { in } L^{1}(Q) \text { strong and a.e. in } Q,
$$

for some $\tau \in L^{1}(Q)$. From (2.110), (2.111) and (2.112) we deduce that

$$
\tau_{n} \rightarrow \tau \quad \text { in } L^{1}(Q) \text { strong and a.e. in } Q .
$$

The strong convergence (2.113) of $\tau_{n}$, the bound (2.76) on $\lambda_{n}$, the convergence (2.77) on $\lambda_{n}\left(x, r_{n}\right)$ and Lebesgue's dominated convergence theorem imply that

$$
\lambda_{n}\left(x, \tau_{n}\right) \rightarrow \lambda(x, \tau) \quad \text { in } L^{p}(Q) \text { strong } \forall p<+\infty \text { and a.e. in } Q .
$$


Using now (2.114) and the weak convergence (2.95) of $\frac{\partial u_{n}}{\partial x}$, we deduce from the definitions (2.99) and (2.101) of $\sigma_{n}$ and $\sigma$ that

$$
\sigma=\lambda(x, \tau)\left(\frac{\partial u}{\partial x}+\frac{\partial \bar{v}}{\partial x}\right)
$$

This proves (2.67). On the other hand, using the weak convergence (2.96) of $\frac{\partial u_{n}}{\partial t}$ implies that

$$
\rho(x) \frac{\partial u_{n}}{\partial t} \rightarrow \rho(x) \frac{\partial u}{\partial t} \quad \text { in } L^{2}(Q) \text { weak. }
$$

Fix now any $w$ in $H_{0}^{1}(\Omega)$ and define $w_{n}$ as its projection on the space generated by the functions $w_{n}^{j}, j=1, \ldots, n-1$, i.e.

$$
w_{n}=\sum_{j=1}^{n-1} W_{n}^{j} w_{n}^{j} .
$$

Multiplying the approximating equation (2.83) by $W_{n}^{i}$, taking the sum from $i=1$ to $i=n-1$ and using the fact that

$$
w_{n} \rightarrow w \text { in } H_{0}^{1}(\Omega) \text { strong, }
$$

one obtains

$$
\left\{\begin{array}{l}
\int_{\Omega} \rho(x) \frac{\partial u}{\partial t} w d x+\int_{\Omega} \lambda(x, \tau)\left(\frac{\partial u}{\partial x}+\frac{\partial \bar{v}}{\partial x}\right) \frac{\partial w}{\partial x} d x= \\
=\int_{\Omega} f w d x-\int_{\Omega} \rho(x) \frac{\partial \bar{v}}{\partial t} w d x \quad \text { in } L^{2}(0, T), \quad \forall w \in H_{0}^{1}(\Omega) .
\end{array}\right.
$$

Equation (2.63) is therefore satisfied.

Fourth step: strong convergence of $\frac{\partial u_{n}}{\partial x}$ in $L^{2}(Q)$

The previous convergences do not allow us to pass to the limit in the right hand side of the approximating equation (2.84). But in the present step we will prove the strong convergence

$$
\frac{\partial u_{n}}{\partial x} \rightarrow \frac{\partial u}{\partial x} \text { in } L^{2}(Q) \text { strongly }
$$

which is nothing but convergence (2.89) of Theorem 2.4.

Multiplication of the approximating equation (2.83) by $u_{n}^{i}(t)$ and addition from $i=1$ to $i=n-1$ yields after integration in time from 0 to $t$

$$
\begin{aligned}
& \frac{1}{2} \int_{\Omega} \rho(x)\left|u_{n}(t)\right|^{2} d x+\int_{0}^{t} \int_{\Omega} \lambda_{n}\left(x, \tau_{n}\right)\left|\frac{\partial u_{n}}{\partial x}+\frac{\partial \bar{v}}{\partial x}\right|^{2} d x d t^{\prime}= \\
& =\frac{1}{2} \int_{\Omega} \rho(x)\left|U_{n}\right|^{2} d x+\int_{0}^{t} \int_{\Omega} \lambda_{n}\left(x, \tau_{n}\right)\left(\frac{\partial u_{n}}{\partial x}+\frac{\partial \bar{v}}{\partial x}\right) \frac{\partial \bar{v}}{\partial x} d x d t^{\prime}+ \\
& +\int_{0}^{t} \int_{\Omega} f u_{n} d x d t^{\prime}-\int_{0}^{t} \int_{\Omega} \rho(x) \frac{\partial \bar{v}}{\partial t} u_{n} d x d t^{\prime}
\end{aligned}
$$


which after another integration in time from 0 to $T$ yields

$$
\left\{\begin{array}{l}
\frac{1}{2} \int_{0}^{T} \int_{\Omega} \rho(x)\left|u_{n}(t)\right|^{2} d x d t+ \\
+\int_{0}^{T} \int_{\Omega}(T-t) \lambda_{n}\left(x, \tau_{n}\right)\left|\frac{\partial u_{n}}{\partial x}+\frac{\partial \bar{v}}{d x}\right|^{2} d x d t= \\
=\frac{1}{2} T \int_{\Omega} \rho(x)\left|U_{n}\right|^{2} d x+ \\
+\int_{0}^{T} \int_{\Omega}(T-t) \lambda_{n}\left(x, \tau_{n}\right)\left(\frac{\partial u_{n}}{\partial x}+\frac{\partial \bar{v}}{d x}\right) \frac{\partial \bar{v}}{\partial x} d x d t+ \\
+\int_{0}^{T} \int_{\Omega}(T-t) f u_{n} d x d t-\int_{0}^{T} \int_{\Omega}(T-t) \rho(x) \frac{\partial \bar{v}}{\partial t} u_{n} d x d t .
\end{array}\right.
$$

The strong convergence (2.80) of $U_{n}$, the weak convergence (2.95) of $u_{n}$ and the strong convergence (2.114) of $\lambda_{n}\left(x, \tau_{n}\right)$ imply that the right hand side of (2.118) converges to

$$
\begin{aligned}
& \frac{1}{2} T \int_{\Omega} \rho(x)\left|u_{0}\right|^{2} d x+\int_{0}^{T} \int_{\Omega}(T-t) \lambda(x, \tau)\left(\frac{\partial u}{\partial x}+\frac{\partial \bar{v}}{\partial x}\right) \frac{\partial \bar{v}}{\partial x} d x d t+ \\
& +\int_{0}^{T} \int_{\Omega}(T-t) f u d x d t-\int_{0}^{T} \int_{\Omega}(T-t) \rho(x) \frac{\partial \bar{v}}{\partial t} u d x d t .
\end{aligned}
$$

On the other hand, the function $u$ belongs to $L^{\infty}\left(0, T ; H_{0}^{1}(\Omega)\right)$ with $\frac{\partial u}{\partial t}$ in $L^{2}\left(0, T ; L^{2}(\Omega)\right)$ (see (2.97)) and satisfies the initial condition (2.98). Using $u$ as test function in equation (2.116) is therefore licit and yields, after integrating twice in time from 0 to $T$

$$
\left\{\begin{array}{l}
\frac{1}{2} \int_{0}^{T} \int_{\Omega} \rho(x)|u|^{2} d x d t+\int_{0}^{T} \int_{\Omega}(T-t) \lambda(x, \tau)\left|\frac{\partial u}{\partial x}+\frac{\partial \bar{v}}{d x}\right|^{2} d x d t= \\
=\frac{1}{2} T \int_{\Omega} \rho(x)\left|u_{0}\right|^{2} d x+\int_{0}^{T} \int_{\Omega}(T-t) \lambda(x, \tau)\left(\frac{\partial u}{\partial x}+\frac{\partial \bar{v}}{\partial x}\right) \frac{\partial \bar{v}}{\partial x} d x d t+ \\
+\int_{0}^{T} \int_{\Omega}(T-t) f u d x d t-\int_{0}^{T} \int_{\Omega}(T-t) \rho(x) \frac{\partial \bar{v}}{\partial t} u d x d t .
\end{array}\right.
$$

Since the right hand side of (2.119) is nothing but the limit of the right hand side of (2.118), and since $u_{n}$ converges to $u$ in $H^{1}(Q)$ weak and therefore in $L^{2}(Q)$ strong (see (2.95) and (2.96)), we have proved that

$$
\left\|\sqrt{(T-t) \lambda_{n}\left(x, \tau_{n}\right)}\left(\frac{\partial u_{n}}{\partial x}+\frac{\partial \bar{v}}{\partial x}\right)\right\|_{L^{2}(Q)}^{2} \rightarrow\left\|\sqrt{(T-t) \lambda(x, \tau)}\left(\frac{\partial u}{\partial x}+\frac{\partial \bar{v}}{\partial x}\right)\right\|_{L^{2}(Q)}^{2} .
$$


But the weak convergence (2.95) of $u_{n}$, combined with the strong convergence (2.114) of $\lambda_{n}\left(x, \tau_{n}\right)$ implies that

$\sqrt{(T-t) \lambda_{n}\left(x, \tau_{n}\right)}\left(\frac{\partial u_{n}}{\partial x}+\frac{\partial \bar{v}}{\partial x}\right) \rightarrow \sqrt{(T-t) \lambda(x, \tau)}\left(\frac{\partial u}{\partial x}+\frac{\partial \bar{v}}{\partial x}\right)$ in $L^{2}(Q)$ weak.

Since the norms converge, the latest convergence is actually strong in $L^{2}(Q)$. Dividing by $\sqrt{\lambda_{n}\left(x, \tau_{n}\right)}$ and using the almost everywhere convergence (2.114) of $\lambda_{n}\left(x, \tau_{n}\right)$ and the lower bound (2.76) on $\lambda_{n}$ implies with the help of Vitali's theorem that

$$
\sqrt{(T-t)} \frac{\partial u_{n}}{\partial x} \rightarrow \sqrt{(T-t)} \frac{\partial u}{\partial x} \text { in } L^{2}(Q) \text { strong. }
$$

Repeating this proof for some $T^{\prime}>T$ proves the strong convergence (2.117).

\section{Fifth step: end of the proof of Theorem 2.4}

In order to complete the proofs of Theorems 2.3 and 2.4 it only remains to prove that (2.62), (2.64), (2.66), (2.71) and (2.91) hold true.

In the previous step we proved that $\frac{\partial u_{n}}{\partial x}$ converges to $\frac{\partial u}{\partial x}$ in $L^{2}(Q)$ strong. With the help of the almost everywhere convergence (2.114) of $\lambda_{n}\left(x, \tau_{n}\right)$, of the upper bound (2.76) on $\lambda_{n}$ and of Vitali's theorem, this implies that

$$
\left|\lambda_{n}\left(x, \tau_{n}\right)\left(\frac{\partial u_{n}}{\partial x}+\frac{\partial \bar{v}}{\partial x}\right)\right|^{2} \rightarrow\left|\lambda(x, \tau)\left(\frac{\partial u}{\partial x}+\frac{\partial \bar{v}}{\partial x}\right)\right|^{2} \quad \text { in } L^{1}(Q) \text { strong. }
$$

The desired results follow from this convergence combined with (2.84), (2.86), (2.100) and (2.113).

\section{Final remark}

The above proof strongly uses the fact that $\Omega=(a, b)$ is one dimensional. This fact was in particular used in a crucial way when studying the approximating stress $\sigma_{n}$ and when proving the strong convergence of $\tau_{n}$ in $L^{1}(Q)$ (second and third steps). The multidimensional case $\Omega \subset \mathbb{R}^{d}$ seems therefore to be out of reach.

\section{Uniqueness, continuity with respect to the data and regularity of the solution}

In this Section, we prove the uniqueness and the local Lipschitz continuity with respect to the data of the solution of (1.1)-(1.5), the existence of which has been proved in Section 2. Under additional hypotheses on the data, we also prove some regularity results, which ensure that the velocity $v$, the temperature $\theta$ and the stress $\sigma$ belong to $L^{\infty}((0, T) \times \Omega)$.

To obtain these results, we make in this Section the same hypotheses as in Section 2, except that we do not assume hypothesis (2.7), namely the fact that $\mu$ is nonincreasing in $s$; in contrast we assume that $\mu$ is Lipschitz continuous in $s$, i.e. that

$$
\left|\mu(x, s)-\mu\left(x, s^{\prime}\right)\right| \leq c_{7}\left|s-s^{\prime}\right| \quad \text { a.e. } x \in \Omega, \forall s, s^{\prime} \in \mathbb{R},
$$


where $0 \leq c_{7}<+\infty$; this hypothesis is stronger than the continuity hypothesis made in (2.1).

\subsection{Statement of the uniqueness and continuity result}

Theorem 3.1 (Uniqueness and continuity with respect to the data) Assume that hypotheses (2.1)-(2.6), (3.1), and (2.8)-(2.12) hold true. Then there exists at most one couple $(v, \theta)$ which satisfies (2.13)-(2.19).

Moreover, if $(v, \theta)$ and $\left(v^{\star}, \theta^{\star}\right)$ are the (unique) solutions of (2.13)-(2.19) for the data $\left(f, v_{a}, v_{b}, v_{0}, \theta_{0}\right)$ and $\left(f^{\star}, v_{a}^{\star}, v_{b}^{\star}, v_{0}^{\star}, \theta_{0}^{\star}\right)$, their difference satisfies

$$
\left\{\begin{array}{l}
\left\|v-v^{\star}\right\|_{L^{\infty}\left(0 ; T ; L^{2}(\Omega)\right)}+\left\|v-v^{\star}\right\|_{L^{2}\left(0, T ; H^{1}(\Omega)\right)}+\left\|\theta-\theta^{\star}\right\|_{L^{\infty}\left(0, T ; L^{1}(\Omega)\right)} \leq \\
\leq C^{\star \star}\left(\left\|f-f^{\star}\right\|_{L^{2}\left(0, T ; L^{2}(\Omega)\right)}+\left\|v_{a}-v_{a}^{\star}\right\|_{H^{1}(0, T)}+\left\|v_{b}-v_{b}^{\star}\right\|_{H^{1}(0, T)}+\right. \\
\left.\quad+\left\|v_{0}-v_{0}^{\star}\right\|_{L^{2}(\Omega)}+\left\|\theta_{0}-\theta_{0}^{\star}\right\|_{L^{1}(\Omega)}\right),
\end{array}\right.
$$

where $C^{\star \star}$ denotes a constant which depends only on $c_{1}, c_{2}, c_{3}, c_{4}, c_{5}, c_{6}, c_{7}$, $(b-a), T$ and $K^{\star}$, when the data satisfy

$$
\left\{\begin{array}{l}
\|f\|_{L^{2}\left(0, T ; L^{2}(\Omega)\right)}+\left\|f^{\star}\right\|_{L^{2}\left(0, T ; L^{2}(\Omega)\right)}+ \\
+\left\|v_{a}\right\|_{H^{1}(0, T)}+\left\|v_{a}^{\star}\right\|_{H^{1}(0, T)}+\left\|v_{b}\right\|_{H^{1}(0, T)}+\left\|v_{b}^{\star}\right\|_{H^{1}(0, T)}+ \\
+\left\|v_{0}\right\|_{H^{1}(\Omega)}+\left\|v_{0}^{\star}\right\|_{H^{1}(\Omega)}+\left\|\theta_{0}\right\|_{L^{1}(\Omega)}+\left\|\theta_{0}^{\star}\right\|_{L^{1}(\Omega)} \leq K^{\star} .
\end{array}\right.
$$

Estimate (3.2) expresses the local Lipschitz continuity of the solution $(v, \theta)$ of (2.13)-(2.19) with respect to the data $\left(f, v_{a}, v_{b}, v_{0}, \theta_{0}\right)$. Observe that the constant $C^{\star \star}$ depends on the norms of the data in the spaces where these data have to be choosen for the existence Theorem 2.1 to hold true, while the Lipschitz continuity takes place in spaces which are slightly different from the spaces where the existence Theorem 2.1 takes place.

As already said after the statement of the existence Theorem 2.1, this uniqueness result implies that the estimates (2.22)-(2.24) on $v, \theta$ and $\sigma$ hold true for every (actually for the unique) solution of (2.13)-(2.19) whenever both hypotheses (3.1) and (2.7) are made.

As far as Neumann's and mixed boundary conditions are concerned, uniqueness and continuity results similar to Theorem 3.1 hold true in the frameworks of Theorems 2.1bis and 2.1ter. We leave their statements and proofs to the reader.

\subsection{A straightforward regularity result for $\tau$}

Before of proving Theorem 3.1, we state and prove a straightforward regularity result on the transformed temperature $\tau$ defined by (2.58). This result will be used in the proof of the uniqueness and continuity Theorem 3.1. 
Proposition 3.1 (Straightforward regularity for $\tau$ ) Assume that hypotheses (2.1)-(2.6) and (2.8)-(2.12) hold true. For every $(u, \tau, \sigma)$ which satisfy (2.61)(2.67), one has

$$
\tau \in W^{1, \infty}\left(0, T ; L^{1}(\Omega)\right) \cap W^{1,1}\left(0, T ; H^{1}(\Omega)\right) \cap H^{1}\left(0, T ; W^{1,1}(\Omega)\right) .
$$

Moreover the following a priori estimates hold true

$$
\|\tau\|_{W^{1, \infty}\left(0, T ; L^{1}(\Omega)\right)}+\|\tau\|_{W^{1,1}\left(0, T ; H^{1}(\Omega)\right)}+\|\tau\|_{H^{1}\left(0, T ; W^{1,1}(\Omega)\right)} \leq E^{\star},
$$

where $E^{\star}$ denotes a constant which depends only on $c_{1}, c_{2}, c_{5}, c_{6}$ and on $R$, when the data satisfy

$$
\|f\|_{L^{2}\left(0, T ; L^{2}(\Omega)\right)}+\left\|v_{a}\right\|_{H^{1}(0, T)}+\left\|v_{b}\right\|_{H^{1}(0, T)}+\left\|u_{0}\right\|_{H_{0}^{1}(\Omega)} \leq R .
$$

Since $W^{1,1}\left(0, T ; H^{1}(\Omega)\right) \subset C^{0}(\bar{Q})$ when $\Omega$ is one dimensional, regularity (3.4) is particular implies that

$$
\tau \in C^{0}(\bar{Q}) \subset L^{\infty}(Q),
$$

and estimates (3.5) imply an estimate on $\tau$ in $L^{\infty}(Q)$.

On the other hand, the original temperature $\theta$ is not as regular as the transformed temperature $\tau$, as far as regularity in $x$ is concerned. Indeed since $\theta$ is defined from $\tau$ by

$$
\theta(t, x)=N(x, \tau(t, x))
$$

(see (2.58) and (2.53)), and since the function $N(x, r)$ is Lipschitz continuous in $r$ (see (2.55)), we have

$$
\frac{\partial \theta}{\partial t}(t, x)=\frac{\partial N}{\partial r}(x, \tau(t, x)) \frac{\partial \tau}{\partial t}(t, x),
$$

and therefore, using (3.4)

$$
\frac{\partial \theta}{\partial t} \in L^{\infty}\left(0, T ; L^{1}(\Omega)\right) \cap L^{2}\left(0, T ; L^{\infty}(\Omega)\right) ;
$$

indeed, since $\frac{\partial N}{\partial r}(x, r)$ only belongs to $L^{\infty}(\Omega)$, we are forced to use the one dimensional embedding $W^{1,1}(\Omega) \subset L^{\infty}(\Omega)$, and we obtain only (3.8). By integration in time this implies that

$$
\theta-\theta_{0} \in W^{1, \infty}\left(0, T ; L^{1}(\Omega)\right) \cap H^{1}\left(0, T ; L^{\infty}(\Omega)\right),
$$

which implies

$$
\theta-\theta_{0} \in C^{0}\left([0, T] ; L^{\infty}(\Omega)\right) \subset L^{\infty}(Q) .
$$

Since $\theta_{0}$ is only assumed to belong to $L^{1}(\Omega)$, assertion (3.9) implies that $\theta \in W^{1, \infty}\left(0, T ; L^{1}(\Omega)\right)$, but does not imply that $\theta \in H^{1}\left(0, T ; L^{\infty}(\Omega)\right)$, and assertion (3.10) does not imply that $\theta \in L^{\infty}(Q)$.

Proof of Proposition 3.1 In view of the regularity (2.68), namely

$$
\sigma \in L^{\infty}\left(0, T ; L^{2}(\Omega)\right) \cap L^{2}\left(0, T ; H^{1}(\Omega)\right),
$$


the equation (2.64) and the initial condition (2.66) on $\tau$, namely

$$
\frac{\partial \tau}{\partial t}=\sigma^{2} \quad \text { in } \mathscr{D}^{\prime}(Q), \quad \tau(0, x)=0 \quad \text { a.e. } x \in \Omega
$$

immediately imply that

$$
\frac{\partial \tau}{\partial t} \in L^{\infty}\left(0, T ; L^{1}(\Omega)\right) \cap L^{1}\left(0, T ; H^{1}(\Omega)\right),
$$

where the latest result follows from the fact that $w \in H^{1}(\Omega)$ implies that $|w|^{2} \in H^{1}(\Omega)$ when $\Omega$ is one dimensional. This proves the two first assertions of (3.4).

The third assertion of (3.4) (as well as the second one) follows from the differentiation of (3.12) with respect to $x$, which yields

$$
\frac{\partial}{\partial t}\left(\frac{\partial \tau}{\partial x}\right)=2 \sigma \frac{\partial \sigma}{\partial x} \quad \text { in } \mathscr{D}^{\prime}(Q), \quad \frac{\partial \tau}{\partial x}(0, x)=0 \quad \text { a.e. } x \in \Omega
$$

and from the regularity (3.11) of $\sigma$, which using $H^{1}(\Omega) \subset L^{\infty}(\Omega)$ implies that

$$
\frac{\partial}{\partial t}\left(\frac{\partial \tau}{\partial x}\right) \in L^{2}\left(0, T ; L^{1}(\Omega)\right) \cap L^{1}\left(0, T ; L^{2}(\Omega)\right) .
$$

Actually differentiation of (3.12) with respect to $x$ is a little bit formal, but the proof can be made rigourous by writing the equation satisfied by the differential quotient $\frac{\tau(t, x+h)-\tau(t, x)}{h}$ and by passing to the limit in $h$.

3.3 Proof of the uniqueness and continuity Theorem 3.1

By the equivalence Theorem 2.2, the uniqueness of the couple $(v, \theta)$ solution of (2.13)-(2.19) is equivalent to the uniqueness of the couple $(u, \tau)$ solution of (2.61)-(2.66). We will therefore prove the uniqueness of the couple $(u, \tau)$.

Observe that in view of the definition (2.59) of $\lambda(x, r)$, of the Lipschitz continuity hypothesis (3.1) on $\mu$ and of the Lipschitz continuity (2.55) of $N(x, r)$, one has

$$
\left|\lambda(x, r)-\lambda\left(x, r^{\prime}\right)\right| \leq \frac{c_{7}}{c_{3} c_{1}}\left|r-r^{\prime}\right| \quad \text { a.e. } x \in \Omega, \forall r, r^{\prime} \in \mathbb{R}
$$

Let $(u, \tau)$ and $\left(u^{\star}, \tau^{\star}\right)$ be two solutions of (2.61)-(2.66) for the data $\left(f, \bar{v}, u_{0}\right)$ and $\left(f^{\star}, \bar{v}^{\star}, u_{0}\right)$. Taking $\left(u-u^{\star}\right)$ as test function in the equations (2.63) satisfied by $u$ and by $u^{\star}$ (this is licit in view of the regularity of $u$ and $u^{\star}$ ), making the difference and using the fact that

$$
\int_{\Omega} \rho(x) \frac{\partial w}{\partial t} w d x=\frac{1}{2} \frac{d}{d t} \int_{\Omega} \rho(x)|w|^{2} d x
$$


for every $w \in H^{1}\left(0, T ; L^{2}(\Omega)\right)$, we obtain after integration in time from 0 to $t$

$$
\left\{\begin{array}{l}
\frac{1}{2} \int_{\Omega} \rho(x)\left|u(t, x)-u^{\star}(t, x)\right|^{2} d x+ \\
+\int_{0}^{t} \int_{\Omega} \lambda\left(x, \tau\left(t^{\prime}, x\right)\right)\left|\frac{\partial u}{\partial x}\left(t^{\prime}, x\right)-\frac{\partial u^{\star}}{\partial x}\left(t^{\prime}, x\right)\right|^{2} d x d t^{\prime}= \\
=\frac{1}{2} \int_{\Omega} \rho(x)\left|u_{0}(x)-u_{0}^{\star}(x)\right|^{2} d x- \\
-\int_{0}^{t} \int_{\Omega}\left(\lambda\left(x, \tau\left(t^{\prime}, x\right)\right)-\lambda\left(x, \tau^{\star}\left(t^{\prime}, x\right)\right)\left(\frac{\partial u^{\star}}{\partial x}\left(t^{\prime}, x\right)+\frac{\partial \bar{v}^{\star}}{\partial x}\left(t^{\prime}, x\right)\right)\right. \\
\quad\left(\frac{\partial u}{\partial x}\left(t^{\prime}, x\right)-\frac{\partial u^{\star}}{\partial x}\left(t^{\prime}, x\right)\right) d x d t^{\prime}- \\
-\int_{0}^{t} \int_{\Omega} \lambda\left(x, \tau\left(t^{\prime}(x)\right)\left(\frac{\partial \bar{v}}{\partial x}\left(t^{\prime}, x\right)-\frac{\partial \bar{v}^{\star}}{\partial x}\left(t^{\prime}, x\right)\right)\right. \\
\left.+\left(\frac{\partial u}{\partial x}\left(t^{\prime}, x\right)-\frac{\partial u^{\star}}{\partial x}\left(t^{\prime}, x\right)\right)\right) d x d t^{\prime}+ \\
+\int_{0}^{t} \int_{\Omega}\left(f\left(t^{\prime}, x\right)-f^{\star}\left(t^{\prime}, x\right)\right)\left(u\left(t^{\prime}, x\right)-u^{\star}\left(t^{\prime}, x\right)\right) d x d t^{\prime}- \\
-\int_{0}^{t} \rho(x)\left(\frac{\partial \bar{v}}{\partial t}\left(t^{\prime}, x\right)-\frac{\partial \bar{v}^{\star}}{\partial t}\left(t^{\prime}, x\right)\right)\left(u\left(t^{\prime}, x\right)-u^{\star}\left(t^{\prime}, x\right)\right) d x d t^{\prime} .
\end{array}\right.
$$

Define the function $\psi^{\star}:(0, T) \rightarrow \mathbb{R}^{+}$by

$$
\psi^{\star}(t)=\sup _{x \in \Omega}\left|\frac{\partial u^{\star}}{\partial x}(t, x)+\frac{\partial \bar{v}^{\star}}{\partial x}(t, x)\right| .
$$

From the definition (2.67) of $\sigma^{\star}$, namely

$$
\sigma^{\star}=\lambda\left(x, \tau^{\star}\right)\left(\frac{\partial u^{\star}}{\partial x}+\frac{\partial \bar{v}^{\star}}{\partial x}\right)
$$

and from the lower bound (2.60) on $\lambda$, we deduce that

$$
\psi^{\star}(t) \leq \frac{1}{c_{1}} \sup _{x \in \Omega}\left|\sigma^{\star}(t, x)\right| .
$$

The regularity (2.68) of $\sigma^{\star}$, namely

$$
\sigma^{\star} \in L^{2}\left(0, T ; H^{1}(\Omega)\right) \subset L^{2}\left(0, T ; L^{\infty}(\Omega)\right),
$$

then implies that

$$
\psi^{\star} \in L^{2}(0, T) .
$$

Here we have used in a crucial way the fact that $\Omega$ is one dimensional. 
Using in the left-hand side of (3.14) the lower bounds (2.6) and (2.60) on $\rho$ and $\lambda$, and in the right-hand side of (3.14) the upper bounds (2.6) and (2.60) on $\rho$ and $\lambda$, the Lipschitz continuity (3.13) of $\lambda$ in $r$, the definition (3.15) of $\psi^{\star}$ and Young's inequality, we obtain for every $t, 0 \leq t \leq T$

$$
\begin{aligned}
& \int \frac{c_{5}}{2} \int_{\Omega}\left|\left(u-u^{\star}\right)(t, x)\right|^{2} d x+c_{1} \int_{0}^{t} \int_{\Omega}\left|\frac{\partial u}{\partial x}-\frac{\partial u^{\star}}{\partial x}\right|^{2} d x d t^{\prime} \leq \\
& \leq \frac{c_{6}}{2} \int_{\Omega}\left|u_{0}(x)-u_{0}^{\star}(x)\right|^{2} d x+ \\
& +\int_{0}^{t} \int_{\Omega} \frac{c_{7}}{c_{3} c_{1}}\left|\tau-\tau^{\star}\right|\left|\frac{\partial u^{\star}}{\partial x}+\frac{\partial \bar{v}^{\star}}{\partial x}\right|\left|\frac{\partial u}{\partial x}-\frac{\partial u^{\star}}{\partial x}\right| d x d t^{\prime}+ \\
& +\int_{0}^{t} \int_{\Omega} c_{2}\left|\frac{\partial \bar{v}}{\partial x}-\frac{\partial \bar{v}^{\star}}{\partial x}\right|\left|\frac{\partial u}{\partial x}-\frac{\partial u^{\star}}{\partial x}\right| d x d t^{\prime}+ \\
& +\int_{0}^{t} \int_{\Omega}\left|f-f^{\star}\right|\left|u-u^{\star}\right| d x d t^{\prime}+ \\
& +\int_{0}^{t} \int_{\Omega} c_{6}\left|\frac{\partial \bar{v}}{\partial t}-\frac{\partial \bar{v}^{\star}}{\partial t}\right|\left|u-u^{\star}\right| d x d t^{\prime} \leq \\
& \leq \frac{c_{6}}{2} \int_{\Omega}\left|u_{0}(x)-u_{0}^{\star}(x)\right|^{2} d x+ \\
& +\frac{c_{1}}{4} \int_{0}^{t} \int_{\Omega}\left|\frac{\partial u}{\partial x}-\frac{\partial u^{\star}}{\partial x}\right|^{2} d x d t^{\prime}+ \\
& +\frac{1}{c_{1}}\left(\frac{c_{7}}{c_{3} c_{1}}\right)^{2} \int_{0}^{t} \int_{\Omega}\left|\psi^{\star}\left(t^{\prime}\right)\right|^{2}\left|\tau-\tau^{\star}\right|^{2} d x d t^{\prime}+ \\
& +\frac{c_{1}}{4} \int_{0}^{t} \int_{\Omega}\left|\frac{\partial u}{\partial x}-\frac{\partial u^{\star}}{\partial x}\right|^{2} d x d t^{\prime}+ \\
& +\frac{1}{c_{1}} c_{2}^{2} \int_{0}^{t} \int_{\Omega}\left|\frac{\partial \bar{v}}{\partial x}-\frac{\partial \bar{v}^{\star}}{\partial x}\right|^{2} d x d t^{\prime}+ \\
& +\frac{c_{5}}{4} \int_{0}^{t} \int_{\Omega}\left|u-u^{\star}\right|^{2} d x d t^{\prime}+\frac{1}{c_{5}} \int_{0}^{t} \int_{\Omega}\left|f-f^{\star}\right|^{2} d x d t^{\prime}+ \\
& +\frac{c_{5}}{4} \int_{0}^{t} \int_{\Omega}\left|u-u^{\star}\right|^{2} d x d t^{\prime}+ \\
& +\frac{1}{c_{5}} c_{6}^{2} \int_{0}^{t} \int_{\Omega}\left|\frac{\partial \bar{v}}{\partial t}-\frac{\partial \bar{v}^{\star}}{\partial t}\right| d x d t^{\prime} .
\end{aligned}
$$


Similarly using the definition (2.67) of $\sigma$ and $\sigma^{\star}$, multiplying by $\left(\tau-\tau^{\star}\right)$ the equations (2.64) satisfied by $\tau$ and by $\tau^{\star}$, integrating in space on $\Omega$ (this is licit since $\tau$ and $\tau^{\star}$ belong to $L^{\infty}(Q)$ by the regularity result (3.7)) and making the difference, we obtain after integration in time from 0 to $t$

$$
\left\{\begin{array}{l}
\frac{1}{2} \int_{\Omega}\left|\tau(t, x)-\tau^{\star}(t, x)\right|^{2} d x= \\
=\int_{0}^{t} \int_{\Omega}\left(\left|\sigma\left(t^{\prime}, x\right)\right|^{2}-\left|\sigma^{\star}\left(t^{\prime}, x\right)\right|^{2}\right)\left(\tau\left(t^{\prime}, x\right)-\tau^{\star}\left(t^{\prime}, x\right)\right) d x d t^{\prime}= \\
=\int_{0}^{t} \int_{\Omega}\left(\sigma\left(t^{\prime}, x\right)-\sigma^{\star}\left(t^{\prime}, x\right)\right) \\
\quad\left(\sigma\left(t^{\prime}, x\right)+\sigma^{\star}\left(t^{\prime}, x\right)\right)\left(\tau\left(t^{\prime}, x\right)-\tau^{\star}\left(t^{\prime}, x\right)\right) d x d t^{\prime}= \\
=\int_{0}^{t} \int_{\Omega}\left(\lambda\left(x, \tau\left(t^{\prime}, x\right)\right)\left(\frac{\partial u}{\partial x}\left(t^{\prime}, x\right)-\frac{\partial u^{\star}}{\partial x}\left(t^{\prime}, x\right)\right)+\right. \\
+\lambda\left(x, \tau\left(t^{\prime}, x\right)\right)\left(\frac{\partial \bar{v}}{\partial x}\left(t^{\prime}, x\right)-\frac{\partial \bar{v}^{\star}}{\partial x}\left(t^{\prime}, x\right)\right)+ \\
\left.+\left(\lambda\left(x, \tau\left(t^{\prime}, x\right)\right)-\lambda\left(x, \tau^{\star}\left(t^{\prime}, x\right)\right)\right)\left(\frac{\partial u^{\star}}{\partial x}\left(t^{\prime}, x\right)+\frac{\partial \bar{v}^{\star}}{\partial x}\left(t^{\prime}, x\right)\right)\right) \\
\left(\sigma\left(t^{\prime}, x\right)+\sigma^{\star}\left(t^{\prime}, x\right)\right)\left(\tau\left(t^{\prime}, x\right)-\tau^{\star}\left(t^{\prime}, x\right)\right) d x d t^{\prime} .
\end{array}\right.
$$

Define the function $\psi:(0, T) \rightarrow \mathbb{R}^{+}$by

$$
\psi(t)=\sup _{x \in \Omega}\left|\sigma(t, x)+\sigma^{\star}(t, x)\right|
$$

Since $\sigma$ and $\sigma^{\star}$ belong to $L^{2}\left(0, T ; H^{1}(\Omega)\right) \subset L^{2}\left(0, T ; L^{\infty}(\Omega)\right)$ (see(2.68)), we obtain

$$
\psi \in L^{2}(0, T)
$$

Here we have again used in a crucial way the fact that $\Omega$ is one dimensional.

Using in (3.19) the upper bound (2.60) on $\lambda$, the Lipschitz continuity (3.13) of $\lambda$ in $r$, the definitions (3.15) and (3.20) of $\psi^{\star}$ and $\psi$ and Young's inequality, we 
obtain for every $t, 0 \leq t \leq T$

$$
\left\{\begin{array}{l}
\frac{1}{2} \int_{\Omega}\left|\left(\tau-\tau^{\star}\right)(t, x)\right|^{2} d x \leq \\
\leq \int_{0}^{t} \int_{\Omega}\left(c_{2}\left|\frac{\partial u}{\partial x}-\frac{\partial u^{\star}}{\partial x}\right|+c_{2}\left|\frac{\partial \bar{v}}{\partial x}-\frac{\partial \bar{v}^{\star}}{\partial x}\right|+\right. \\
\left.\quad+\frac{c_{7}}{c_{3} c_{1}}\left|\tau-\tau^{\star}\right| \frac{\partial u^{\star}}{\partial x}+\frac{\partial \bar{v}^{\star}}{\partial x} \mid\right) \psi\left(t^{\prime}\right)\left|\tau-\tau^{\star}\right| d x d t^{\prime} \leq \\
\leq \frac{c_{1}}{4} \int_{0}^{t} \int_{\Omega}\left|\frac{\partial u}{\partial x}-\frac{\partial u^{\star}}{\partial x}\right|^{2} d x d t^{\prime}+\frac{c_{2}^{2}}{c_{1}} \int_{0}^{t} \int_{\Omega}\left|\psi\left(t^{\prime}\right)\right|^{2}\left|\tau-\tau^{\star}\right|^{2} d x d t^{\prime}+ \\
+\frac{c_{2}}{2} \int_{0}^{t} \int_{\Omega}\left|\frac{\partial \bar{v}}{\partial x}-\frac{\partial \bar{v}^{\star}}{\partial x}\right|^{2} d x d t^{\prime}+\frac{c_{2}}{2} \int_{0}^{t} \int_{\Omega}\left|\psi\left(t^{\prime}\right)\right|^{2}\left|\tau-\tau^{\star}\right|^{2} d x d t^{\prime}+ \\
+\frac{c_{7}}{c_{3} c_{1}} \int_{0}^{t} \int_{\Omega} \psi^{\star}\left(t^{\prime}\right) \psi\left(t^{\prime}\right)\left|\tau-\tau^{\star}\right|^{2} d x d t^{\prime}
\end{array}\right.
$$

Adding (3.18) and (3.22) yields for every $t, 0 \leq t \leq T$

$$
\left\{\begin{array}{l}
\frac{c_{5}}{2} \int_{\Omega}\left|\left(u-u^{\star}\right)(t, x)\right|^{2} d x+\frac{c_{1}}{4} \int_{0}^{t} \int_{\Omega}\left|\frac{\partial u}{\partial x}-\frac{\partial u^{\star}}{\partial x}\right|^{2} d x d t^{\prime}+ \\
+\frac{1}{2} \int_{\Omega}\left|\left(\tau-\tau^{\star}\right)(t, x)\right|^{2} d x \leq \\
\leq \frac{c_{6}}{2} \int_{\Omega}\left|u_{0}(x)-u_{0}^{\star}(x)\right|^{2} d x+ \\
+\left(\frac{c_{2}^{2}}{c_{1}}+\frac{c_{6}^{2}}{c_{5}}+\frac{c_{2}}{2}\right) \int_{0}^{t} \int_{\Omega}\left|\frac{\partial \bar{v}}{\partial x}-\frac{\partial \bar{v}^{\star}}{\partial x}\right|^{2} d x d t^{\prime}+ \\
+\frac{1}{c_{5}} \int_{0}^{t} \int_{\Omega}\left|f-f^{\star}\right|^{2} d x d t^{\prime}+ \\
+\frac{c_{5}}{2} \int_{0}^{t} \int_{\Omega}\left|u-u^{\star}\right|^{2} d x d t^{\prime}+ \\
+\int_{0}^{t}\left(\frac{c_{7}^{2}}{c_{3}^{2} c_{1}^{3}}\left|\psi^{\star}\left(t^{\prime}\right)\right|^{2}+\left(\frac{c_{2}^{2}}{c_{1}}+\frac{c_{2}}{2}\right)\left|\psi^{2}\left(t^{\prime}\right)\right|^{2}+\frac{c_{7}}{c_{3} c_{1}} \psi^{\star}\left(t^{\prime}\right) \psi\left(t^{\prime}\right)\right) \\
\int_{\Omega}\left|\tau-\tau^{\star}\right|^{2} d x d t^{\prime} .
\end{array}\right.
$$


Define the functions $\varphi:(0, T) \rightarrow \mathbb{R}^{+}, \delta:(0, T) \rightarrow \mathbb{R}^{+}$and $\gamma:(0, T) \rightarrow \mathbb{R}^{+}$by

$$
\begin{aligned}
\varphi(t) & =\frac{c_{5}}{2} \int_{\Omega}\left|\left(u-u^{\star}\right)(t, x)\right|^{2} d x+\frac{c_{1}}{4} \int_{0}^{t} \int_{\Omega}\left|\left(\frac{\partial u}{\partial x}-\frac{\partial u^{\star}}{\partial x}\right)\left(t^{\prime}, x\right)\right|^{2} d x d t^{\prime}+ \\
& +\frac{1}{2} \int_{\Omega}\left|\left(\tau-\tau^{\star}\right)(t, x)\right|^{2} d x \\
\delta(t) & =\frac{c_{6}}{2} \int_{\Omega}\left|u_{0}(x)-u_{0}^{\star}(x)\right|^{2} d x+ \\
& +\left(\frac{c_{2}^{2}}{c_{1}}+\frac{c_{6}^{2}}{c_{5}}+\frac{c_{2}}{2}\right) \int_{0}^{t} \int_{\Omega}\left|\left(\frac{\partial \bar{v}}{\partial x}-\frac{\partial \bar{v}^{\star}}{\partial x}\right)\left(t^{\prime}, x\right)\right|^{2} d x d t^{\prime}+ \\
& +\frac{1}{c_{5}} \int_{0}^{t} \int_{\Omega}\left|\left(f-f^{\star}\right)\left(t^{\prime}, x\right)\right|^{2} d x d t^{\prime}, \\
\gamma(t) & =1+2\left(\frac{c_{7}^{2}}{c_{3}^{2} c_{1}^{3}}\left|\psi^{\star}(t)\right|^{2}+\left(\frac{c_{2}^{2}}{c_{1}}+\frac{c_{2}}{2}\right)\left|\psi^{2}(t)\right|^{2}+\frac{c_{7}}{c_{3} c_{1}} \psi^{\star}(t) \psi(t)\right) .
\end{aligned}
$$

Note that the function $\varphi$ belongs to $L^{\infty}(0, T)$ in view of (2.61) and (3.7), that the function $\delta$ belongs to $L^{\infty}(0, T)$ in view of (2.8), (2.9), (2.10), (2.43) and (2.44), and that the function $\gamma$ belongs to $L^{1}(0, T)$ in view of (3.17) and (3.21).

Since $\frac{c_{5}}{2} \int_{\Omega}\left|\left(u-u^{\star}\right)\left(t^{\prime}, x\right)\right|^{2} d x \leq \varphi\left(t^{\prime}\right)$ and since $\int_{\Omega}\left|\left(\tau-\tau^{\star}\right)\left(t^{\prime}, x\right)\right|^{2} d x \leq$ $\leq 2 \varphi\left(t^{\prime}\right)$, inequality (3.23) implies that for every $t, 0 \leq t \leq T$

$$
\varphi(t) \leq \delta(t)+\int_{0}^{t} \gamma\left(t^{\prime}\right) \varphi\left(t^{\prime}\right) d t^{\prime}
$$

Setting $\phi(t)=\int_{0}^{t} \gamma\left(t^{\prime}\right) \varphi\left(t^{\prime}\right) d t^{\prime}$ and observing that $\phi(0)=0$ and that $\phi^{\prime}=\gamma \varphi \leq$ $\leq \gamma \delta+\gamma \phi$, an easy computation shows that every solution $\varphi$ of inequality (3.24) satisfies for every $t, 0 \leq t \leq T$

$$
\varphi(t) \leq \delta(t)+\int_{0}^{t} \delta\left(t^{\prime}\right) \gamma\left(t^{\prime}\right)\left(\exp \int_{t^{\prime}}^{t} \gamma(s) d s\right) d t^{\prime} .
$$

When the data coincide, i.e. when $\left(f, \bar{v}, u_{0}\right)=\left(f^{\star}, \bar{v}^{\star}, u_{0}^{\star}\right)$, then $\delta(t)=0$ for every $t, 0 \leq t \leq T$, and (3.25) implies that $\varphi(t)=0$ for every $t, 0 \leq t \leq T$. This proves the uniqueness of the solution $(u, \tau)$ of $(2.61)-(2.66)$, and therefore the uniqueness of the solution $(v, \theta)$ of (2.13)-(2.19).

But (3.25) also proves the local Lipschitz continuity of the solution $(u, \tau)$ of (2.61)-(2.66) with respect to the data. Indeed using the definitions of $\varphi, \delta$, the definition (2.43) of $\bar{v}$ and $\bar{v}^{\star}$ and the fact that for every $t, 0 \leq t \leq T$

$$
\begin{aligned}
\delta(t) & +\int_{0}^{t} \delta\left(t^{\prime}\right) \gamma\left(t^{\prime}\right)\left(\exp \int_{t^{\prime}}^{t} \gamma(s) d s\right) d t^{\prime} \leq \\
& \leq\|\delta\|_{L^{\infty}(0, T)}\left(1+\|\gamma\|_{L^{1}(0, T)} \exp \left(\|\gamma\|_{L^{1}(0, T)}\right)\right),
\end{aligned}
$$


inequality (3.25) implies that

$$
\left\{\begin{array}{l}
\left\|u-u^{\star}\right\|_{L^{\infty}\left(0, T ; L^{2}(\Omega)\right)}^{2}+\left\|u-u^{\star}\right\|_{L^{2}\left(0, T ; H_{0}^{1}(\Omega)\right)}^{2}+\left\|\tau-\tau^{\star}\right\|_{L^{\infty}\left(0, T ; L^{2}(\Omega)\right)}^{2} \leq \\
\leq C^{\star \star \star}\left(\left\|f-f^{\star}\right\|_{L^{2}\left(0, T ; L^{2}(\Omega)\right)}^{2}+\left\|v_{a}-v_{a}^{\star}\right\|_{L^{2}(0, T)}^{2}+\left\|v_{b}-v_{b}^{\star}\right\|_{L^{2}(0, T)}+\right. \\
\left.\quad+\left\|u_{0}-u_{0}^{\star}\right\|_{L^{2}(\Omega)}^{2}\right),
\end{array}\right.
$$

where the constant $C^{\star \star \star}$ depends only on $c_{1}, c_{2}, c_{3}, c_{5}, c_{6}, c_{7},(b-a)$ and on the norms of $\psi^{\star}$ and $\psi$ in $L^{2}(0, T)$; by the definitions (3.15) and (3.20) of $\psi^{\star}$ and $\psi$, the property (3.16) and the a priori estimate (2.72), these norms depend only on $c_{1}, c_{2}, c_{5}, c_{6}$ and $L$, where $L$ is defined by (2.73).

Estimate (3.26) expresses the local Lipschitz continuity of the solution $(u, \tau)$ of (2.61)-(2.66) with respect to the data $\left(f, v_{a}, v_{b}, u_{0}\right)$. Observe that the constant $C^{\star \star \star}$ depends on the norms of the data in the spaces where these data have to be choosen for the existence Theorem 2.3 of a solution of the transformed system to hold true, while the Lipschitz continuity takes place in spaces which are slightly different from the spaces where the existence Theorem 2.3 takes place.

Let us now pass to the local Lipschitz continuity of $(v, \theta)$ with respect to the data. In view of the definition (2.45) of $u$ and $u^{\star}$, namely

$$
v=u+\bar{v}, \quad v^{\star}=u^{\star}+\bar{v}^{\star},
$$

we have, since $H^{1}(0, T) \subset L^{\infty}(0, T)$

$$
\left\{\begin{array}{l}
\left\|v-v^{\star}\right\|_{L^{\infty}\left(0, T ; L^{2}(\Omega)\right) \cap L^{2}\left(0, T ; H^{1}(\Omega)\right)} \leq \\
\leq\left\|u-u^{\star}\right\|_{L^{\infty}\left(0, T ; L^{2}(\Omega)\right) \cap L^{2}\left(0, T ; H^{1}(\Omega)\right)}+ \\
\quad+\left\|\bar{v}-\bar{v}^{\star}\right\|_{L^{\infty}\left(0, T ; L^{2}(\Omega)\right) \cap L^{2}\left(0, T ; H^{1}(\Omega)\right)} \leq \\
\leq\left\|u-u^{\star}\right\|_{L^{\infty}\left(0, T ; L^{2}(\Omega)\right) \cap L^{2}\left(0, T ; H^{1}(\Omega)\right)}+ \\
\quad+\left(C(T) \sqrt{b-a}+\frac{1}{b-a}\right)\left(\left\|v_{a}-v_{a}^{\star}\right\|_{H^{1}(0, T)}+\left\|v_{b}-v_{b}^{\star}\right\|_{H^{1}(0, T)}\right),
\end{array}\right.
$$

where the constant $C(T)$ depends only on $T$.

On the other hand, the definition (2.52) of $M$ and $M^{\star}$ implies that

$$
M(x, s)-M^{\star}(x, s)=\int_{\theta_{0}(x)}^{\theta_{0}^{\star}(x)} c\left(x, s^{\prime}\right) \mu\left(x, s^{\prime}\right) d s^{\prime} \quad \text { a.e. } x \in \Omega, \forall s \in \mathbb{R}
$$


and therefore

$$
\left\{\begin{array}{l}
M(x, \theta(t, x))-M^{\star}\left(x, \theta^{\star}(t, x)\right)= \\
\quad=M(x, \theta(t, x))-M\left(x, \theta^{\star}(t, x)\right)+ \\
\quad+M\left(x, \theta^{\star}(t, x)\right)-M^{\star}\left(x, \theta^{\star}(t, x)\right)= \\
\quad=M(x, \theta(t, x))-M\left(x, \theta^{\star}(t, x)\right)+\int_{\theta_{0}(x)}^{\theta_{0}^{\star}(x)} c\left(x, s^{\prime}\right) \mu\left(x, s^{\prime}\right) d s^{\prime} .
\end{array}\right.
$$

Using the first inequality of (2.54), namely

$$
\left|s-s^{\prime}\right| \leq \frac{1}{c_{3} c_{1}}\left|M(x, s)-M\left(x, s^{\prime}\right)\right| \quad \text { a.e. } x \in \Omega, \forall s, s^{\prime} \in \mathbb{R},
$$

equality (3.28) and the definition (2.58) of $\tau$ and $\tau^{\star}$, namely

$$
M(x, \theta(t, x))-M^{\star}\left(x, \theta^{\star}(t, x)\right)=\tau(t, x)-\tau^{\star}(t, x),
$$

and finally the lower bounds (2.4) and (2.5) on $\mu$ and $c$, we obtain

$$
\left\{\begin{array}{r}
\left|\theta(t, x)-\theta^{\star}(t, x)\right| \leq \frac{1}{c_{3} c_{1}}\left(\left|\tau(t, x)-\tau^{\star}(t, x)\right|+c_{4} c_{2}\left|\theta_{0}(x)-\theta_{0}^{\star}(x)\right|\right) \\
\text { a.e. } x \in \Omega, t \in(0, T),
\end{array}\right.
$$

which implies that

$$
\left\{\begin{array}{l}
\left\|\theta-\theta^{\star}\right\|_{L^{\infty}\left(0, T ; L^{1}(\Omega)\right)} \leq \\
\quad \leq \frac{1}{c_{3} c_{1}}\left\|\tau-\tau^{\star}\right\|_{L^{\infty}\left(0, T ; L^{1}(\Omega)\right)}+\frac{c_{4} c_{2}}{c_{3} c_{1}}\left\|\theta_{0}-\theta_{0}^{\star}\right\|_{L^{1}(\Omega)} .
\end{array}\right.
$$

Combining estimates (3.27) and (3.30) with the local Lipschitz continuity (3.26) of $(u, \tau)$ with respect to the data and with the definition (2.44) of $u_{0}$ and $u_{0}^{\star}$ completes the proof of estimate (3.2) and of Theorem 3.1.

3.4 Regularity of $\frac{\partial v}{\partial t}$

The goal of this Subsection is to prove the $L^{2}\left(0, T ; H^{1}(\Omega)\right) \cap L^{\infty}\left(0, T ; L^{2}(\Omega)\right)$ regularity of $\frac{\partial v}{\partial t}$, a result which has important consequences on the regularity of the solution of (2.13)-(2.19). We will make these consequences explicit in Theorem 3.3 below (see Subsection 3.5 below).

Theorem 3.2 (Regularity of $\frac{\partial v}{\partial t}$ ) Assume that hypotheses (2.1)-(2.6), (3.1) and (2.8)-(2.12) hold true, and further that the data $f, v_{a}, v_{b}$ and $\theta_{0}$ satisfy

$$
\frac{\partial f}{\partial t} \in L^{2}\left(0, T ; H^{-1}(\Omega)\right),
$$




$$
\begin{gathered}
v_{a} \in H^{2}(0, T), \quad v_{b} \in H^{2}(0, T), \\
f(0, x)+\frac{\partial}{\partial x}\left(\mu\left(x, \theta_{0}(x)\right) \frac{\partial v_{0}}{\partial x}\right) \in L^{2}(\Omega) .
\end{gathered}
$$

Then every solution $(v, \theta)$ of (2.13)-(2.19) satisfies

$$
\frac{\partial v}{\partial t} \in L^{\infty}\left(0, T ; L^{2}(\Omega)\right) \cap L^{2}\left(0, T ; H^{1}(\Omega)\right),
$$

and the following a priori estimates hold true

$$
\left\|\frac{\partial v}{\partial t}\right\|_{L^{\infty}\left(0, T ; L^{2}(\Omega)\right)}+\left\|\frac{\partial v}{\partial t}\right\|_{L^{2}\left(0, T ; H^{1}(\Omega)\right)} \leq F^{\star}
$$

where $F^{\star}$ denotes a constant which depends only on $c_{1}, c_{2}, c_{3}, c_{4}, c_{5}, c_{6}, c_{7},(b-a)$ and $S$, when the data satisfy

$$
\left\{\begin{array}{l}
\|f\|_{L^{2}\left(0, T ; L^{2}(\Omega)\right)}+\left\|\frac{\partial f}{\partial t}\right\|_{L^{2}\left(0, T ; H^{-1}(\Omega)\right)}+ \\
+\left\|v_{a}\right\|_{H^{2}(0, T)}+\left\|v_{b}\right\|_{H^{2}(0, T)}+\left\|v_{0}\right\|_{H^{1}(\Omega)}+\left\|\theta_{0}\right\|_{L^{1}(\Omega)}+ \\
+\left\|f(0)+\frac{\partial}{\partial x}\left(\mu\left(x, \theta_{0}\right) \frac{\partial v_{0}}{\partial x}\right)\right\|_{L^{2}(\Omega)} \leq S
\end{array}\right.
$$

Note that the compatibility condition (3.33) makes sense: indeed $f$ satisfies $f \in L^{2}\left(0, T ; L^{2}(\Omega)\right)$ with $\frac{\partial f}{\partial t} \in L^{2}\left(0, T ; H^{-1}(\Omega)\right)$, and therefore $f$ belongs to $\left.C^{0}([0, T]), H^{-1}(\Omega)\right)$, which allows one to define $f(0)$ as an element of $H^{-1}(\Omega)$; on the other hand $\frac{\partial}{\partial x}\left(\mu\left(x, \theta_{0}\right) \frac{\partial v_{0}}{\partial x}\right)$ belongs to $H^{-1}(\Omega)$. Therefore hypothesis (3.33) asserts that the sum of those two terms of $H^{-1}(\Omega)$ actually belongs to $L^{2}(\Omega)$.

Note also that under hypotheses of Theorem 3.2, which are more restrictive than those of the uniqueness Theorem 3.1, the solution $(v, \theta)$ of $(2.13)-(2.19)$, if it exists, is unique. Therefore the assertion "Then every solution $(v, \theta)$ of (2.13)(2.19) satisfies (3.34)" can as well be written as "Then the unique solution $(v, \theta)$ of (2.13)-(2.19), if it exists, satisfies (3.34)".

As far as Neumann's and mixed boundary conditions are concerned, regularity results similar to Theorem 3.2 hold true in the frameworks of Theorem 2 .1bis and 2.1ter. We leave their statements and proofs to the reader. Let us just mention that the statement (3.32) has to be replaced by

$$
\sigma_{a} \in H^{1}(0, T), \quad \sigma_{b} \in H^{1}(0, T),
$$

in the case where Neumann's boundary conditions are concerned, or by

$$
v_{a} \in H^{2}(0, T), \quad \sigma_{b} \in H^{1}(0, T),
$$


in the case where mixed boundary conditions are concerned, and that in both cases that the statement (3.31) has to be replaced by

$$
\frac{\partial f}{\partial t} \in L^{2}\left(0, T ; L^{2}(\Omega)\right)
$$

while the statement (3.33) has to be kept.

Proof of Theorem 3.2 Since $u$ and $v$ are linked by the relation (2.45), namely

$$
u=v-\bar{v}, \quad \text { where } \quad \bar{v}(t, x)=\frac{(x-a) v_{b}(t)+(b-x) v_{a}(x)}{b-a},
$$

(see (2.43) for the definition of $\bar{v}$ ), it is equivalent to prove Theorem 3.2 or to prove that the solution $(u, \tau)$ of (2.61)-(2.66) satisfies

$$
\frac{\partial u}{\partial t} \in L^{2}\left(0, T ; H_{0}^{1}(\Omega)\right) \cap L^{\infty}\left(0, T ; L^{2}(\Omega)\right),
$$

and the corresponding a priori estimate.

\section{First step: a formal proof}

We begin with a formal proof of (3.37) which gives the idea of the proof.

Define

$$
w=\frac{\partial u}{\partial t} .
$$

Differentiating equation (2.63) on $u$ with respect to $t$, we formally obtain

$$
\left\{\begin{array}{l}
\rho(x) \frac{\partial w}{\partial t}-\frac{\partial}{\partial x}\left(\lambda(x, \tau) \frac{\partial w}{\partial x}\right)= \\
=\frac{\partial}{\partial x}\left(\lambda(x, \tau) \frac{\partial^{2} \bar{v}}{\partial t \partial x}\right)+\frac{\partial}{\partial x}\left(\frac{\partial \lambda}{\partial r}(x, \tau) \frac{\partial \tau}{\partial t}\left(\frac{\partial u}{\partial x}+\frac{\partial \bar{v}}{\partial x}\right)\right)+ \\
+\frac{\partial f}{\partial t}-\rho(x) \frac{\partial^{2} \bar{v}}{\partial t^{2}} \quad \text { in } Q .
\end{array}\right.
$$

In view of equation (2.64) on $\tau$ and of the definition (2.67) of $\sigma$ one has

$$
\frac{\partial \lambda}{\partial r}(x, \tau) \frac{d \tau}{d t}\left(\frac{\partial u}{\partial x}+\frac{\partial \bar{v}}{\partial x}\right)=\frac{\partial \lambda}{\partial r}(x, \tau) \frac{1}{\lambda(x, \tau)} \sigma^{3}
$$

which belongs to $L^{2}(Q)$ in view of the Lipschitz continuity (3.13) of $\lambda$ in $r$, of the lower bound (2.60) on $\lambda$ and of the regularity (2.68) of $\sigma$ combined with the result (3.69) of Lemma 3.1 below, which implies that $\sigma$ belongs to $L^{6}(Q)$. On the other hand, by hypotheses (3.31) and (3.32) on $f, v_{a}$ and $v_{b}$, the three other terms of the right hand side of (3.39) belong to $L^{2}\left(0, T ; H^{-1}(\Omega)\right)$. Therefore $w$ satisfies

$$
\rho(x) \frac{\partial w}{\partial t}-\frac{\partial}{\partial x}\left(\lambda(x, \tau) \frac{\partial w}{\partial x}\right)=g \quad \text { in } Q
$$


where $g \in L^{2}\left(0, T ; H^{-1}(\Omega)\right)$. Moreover, since $u(t, a)=u(t, b)=0$ for every $t \in(0, T), w=\frac{\partial u}{\partial t}$ satisfies the same homogeneous boundary conditions, i.e.

$$
w(t, a)=w(t, b)=0 \quad \forall t \in(0, T) .
$$

Finally equation (2.63) taken for $t=0$ formally implies that

$$
\begin{aligned}
& \rho(x) w(0, x)=\rho(x) \frac{\partial u}{\partial t}(0, x)= \\
& =\frac{\partial}{\partial x}\left(\lambda(x, \tau(0, x))\left(\frac{\partial u}{\partial x}+\frac{\partial \bar{v}}{\partial x}\right)(0, x)\right)+f(0, x)-\rho(x) \frac{\partial \bar{v}}{\partial t}(0, x),
\end{aligned}
$$

whose right-hand side belongs to $L^{2}(\Omega)$ in view of the fact that $\frac{\partial \bar{v}}{\partial t}(0, x)$ belongs to $L^{2}(\Omega)$, of the compatibility condition (3.33), of the initial conditions (2.65) and (2.66) on $u$ and $\tau$, of the definitions (2.44) and (2.59) of $u_{0}$ and $\lambda$ and of property (2.57).

Therefore equation (3.40) on $w$ and its boundary conditions (3.41) have to be completed by the initial condition

$$
w(0, x)=w_{0}(x),
$$

where $w_{0}$ belongs to $L^{2}(\Omega)$. This formally implies the desired regularity (3.37) on $\frac{\partial u}{\partial t}=w$.

\section{Second step: an abstract result}

To make the above proof correct, we can for example proceed as in the two following steps. Note that the proof of the present step is not restricted to the case where $\Omega$ is one dimensional.

If $(u, \tau)$ is any solution of (2.61)-(2.66), one deduces from (2.61), (2.63) and (2.65) that $u$ is the (unique) solution of the problem

$$
\left\{\begin{array}{l}
u \in L^{2}\left(0, T ; H_{0}^{1}(\Omega)\right) \cap H^{1}\left(0, T ; L^{2}(\Omega)\right), \\
\rho(x) \frac{\partial u}{\partial t}-\frac{\partial}{\partial x}\left(a(t, x) \frac{\partial u}{\partial x}\right)=h \quad \text { in } \mathscr{D}^{\prime}(Q), \\
u(0, x)=u_{0}(x) \quad \text { a.e. } x \in \Omega,
\end{array}\right.
$$

where $a$ and $h$ are defined by

$$
\begin{gathered}
a(t, x)=\lambda(x, \tau(t, x)), \\
h(t, x)=f(t, x)-\rho(x) \frac{\partial \bar{v}}{\partial t}(t, x)+\frac{\partial}{\partial x}\left(a(t, x) \frac{\partial \bar{v}}{\partial x}(t, x)\right) .
\end{gathered}
$$

Since $a$ belongs to $L^{\infty}(Q)$ with $a(t, x) \geq c_{1}>0$, since $h$ belongs to $L^{2}\left(0, T ; H^{-1}(\Omega)\right)$, since $\rho$ belongs to $L^{\infty}(\Omega)$ with $\rho(x) \geq c_{5}>0$ and since $u_{0}$ 
belongs to $L^{2}(\Omega)$, the solution of (3.42) is unique. Moreover if one considers a Galerkin's basis $y^{1}, y^{2}, \ldots$ of $H_{0}^{1}(\Omega)$, the solution $u_{n}$ of the approximating problem

$$
u_{n}(t, x)=\sum_{j=1}^{n} u_{n}^{j}(t) y^{j}(x)
$$

$$
\left\{\begin{array}{l}
u_{n} \in H^{1}\left(0, T ; H_{0}^{1}(\Omega)\right) \\
\int_{\Omega} \rho \frac{\partial u_{n}}{\partial t} y^{i} d x+\int_{\Omega} a(t, x) \frac{\partial u_{n}}{\partial x} \frac{\partial y^{i}}{\partial x} d x={ }_{H^{-1}(\Omega)}\left\langle h(t), y^{i}\right\rangle_{H_{0}^{1}(\Omega)} \\
\int_{\Omega} u_{n}(0, x) y^{i} d x=\int_{\Omega} u_{0}(x) y^{i} d x \\
i=1,2, \ldots, n,
\end{array}\right.
$$

(where the functions $u_{n}^{j} \in H^{1}(0, T), j=1,2, \ldots, n$, are the very unknowns of the approximating problem (3.45)) satisfies

$$
u_{n} \rightarrow u \quad \text { in } L^{2}\left(0, T ; H_{0}^{1}(\Omega)\right) \text { strong. }
$$

Define $w_{n}$ by

$$
w_{n}=\frac{\partial u_{n}}{\partial t}=\sum_{j=1}^{n} \frac{\partial u_{n}^{j}}{\partial t}(t) y^{j}(x)
$$

If we assume that

$$
\begin{aligned}
& \frac{\partial h}{\partial t} \in L^{2}\left(0, T ; H^{-1}(\Omega)\right), \\
& \frac{\partial a}{\partial t} \in L^{2}\left(0, T ; L^{\infty}(\Omega)\right),
\end{aligned}
$$

it is now licit to differentiate with respect to $t$ the equation in (3.45). We obtain that $w_{n}$ satisfies

$$
\left\{\begin{array}{l}
\int_{\Omega} \rho \frac{\partial w_{n}}{\partial t} y^{i} d x+\int_{\Omega} a(t, x) \frac{\partial w_{n}}{\partial x} \frac{\partial y^{i}}{\partial x} d x= \\
={ }_{H^{-1}(\Omega)}\left\langle\frac{\partial h}{\partial t}(t), y^{i}\right\rangle_{H_{0}^{1}(\Omega)}-\int_{\Omega} \frac{\partial a}{\partial t}(t, x) \frac{\partial u_{n}}{\partial x} \frac{\partial y^{i}}{\partial x} d x, \\
i=1,2, \ldots, n,
\end{array}\right.
$$

which implies that $w_{n}$ belongs to $H^{1}\left(0, T ; H_{0}^{1}(\Omega)\right)$.

For $t=0$ the equation in (3.45) yields

$$
\left\{\begin{aligned}
\int_{\Omega} \rho w_{n}(0) y^{i} d x & ={ }_{H^{-1}(\Omega)}<h(0), y^{i}>_{H_{0}^{1}(\Omega)}-\int_{\Omega} a(0, x) \frac{\partial u_{0}}{\partial x} \frac{\partial y^{i}}{\partial x} d x= \\
& ={ }_{H^{-1}(\Omega)}<h(0)+\frac{\partial}{\partial x}\left(a(0, x) \frac{\partial u_{0}}{\partial x}\right), y^{i}>_{H_{0}^{1}(\Omega)}, \\
i=1,2, \ldots, n . &
\end{aligned}\right.
$$


Multiplication of (3.51) by $\frac{d u_{n}^{i}}{d t}(0)$ and addition from $i=1$ to $i=n$ yields

$$
\int_{\Omega} \rho\left|w_{n}(0)\right|^{2} d x={ }_{H^{-1}(\Omega)}\left\langle h(0)+\frac{\partial}{\partial x}\left(a(0, x) \frac{\partial u_{0}}{\partial x}\right), w_{n}(0)\right\rangle_{H_{0}^{1}(\Omega)} .
$$

If we assume that

$$
h(0)+\frac{\partial}{\partial x}\left(a(0, x) \frac{\partial u_{0}}{\partial x}\right) \in L^{2}(\Omega),
$$

this implies with the help of the lower bound (2.6) on $\rho$ that

$$
\left\|w_{n}(0)\right\|_{L^{2}(\Omega)} \leq \frac{1}{c_{5}}\left\|h(0)+\frac{\partial}{\partial x}\left(a(0, x) \frac{\partial u_{0}}{\partial x}\right)\right\|_{L^{2}(\Omega)} .
$$

Multiplication of (3.50) by $\frac{d u_{n}^{i}}{d t}(t)$, addition from $i=1$ to $i=n$, integration in time from 0 to $t$, then use of the lower bound (2.6) on $\rho$, of the coerciveness of $a$, of the bound (3.53) and of Young's inequality yields for every $t, 0<t \leq T$

$$
\begin{aligned}
& \frac{1}{2} c_{5} \int_{\Omega}\left|w_{n}(t, x)\right|^{2} d x+c_{1} \int_{0}^{t} \int_{\Omega}\left|\frac{\partial w_{n}}{\partial x}\left(t^{\prime}, x\right)\right|^{2} d x d t^{\prime} \leq \\
& \leq \frac{1}{2} \int_{\Omega} \rho\left|w_{n}(0, x)\right|^{2} d x+\int_{0}^{t}\left\|\frac{\partial h}{\partial t}\left(t^{\prime}\right)\right\|_{H^{-1}(\Omega)}\left\|w_{n}\left(t^{\prime}\right)\right\|_{H_{0}^{1}(\Omega)} d t^{\prime}+ \\
& +\int_{0}^{t}\left\|\frac{\partial a}{\partial t}\left(t^{\prime}\right)\right\|_{L^{\infty}(\Omega)}\left\|\frac{\partial u_{n}}{\partial x}\left(t^{\prime}\right)\right\|_{L^{2}(\Omega)}\left\|\frac{\partial w_{n}}{\partial x}\left(t^{\prime}\right)\right\|_{L^{2}(\Omega)} d t^{\prime} \leq \\
& \leq \frac{1}{2} \frac{c_{6}}{c_{5}^{2}}\left\|h(0)+\frac{\partial}{\partial x}\left(a(0, x) \frac{\partial u_{0}}{\partial x}\right)\right\|_{L^{2}(\Omega)}^{2}+ \\
& +\frac{c_{1}}{4} \int_{0}^{t}\left\|w_{n}\left(t^{\prime}\right)\right\|_{H_{0}^{1}(\Omega)}^{2} d t^{\prime}+\frac{1}{c_{1}}\left\|\frac{\partial h}{\partial t}\right\|_{L^{2}\left(0, T ; H^{-1}(\Omega)\right)}^{2}+ \\
& +\frac{c_{1}}{4} \int_{0}^{t}\left\|\frac{\partial w_{n}}{\partial x}\left(t^{\prime}\right)\right\|_{L^{2}(\Omega)}^{2} d t^{\prime}+\frac{1}{c_{1}} \int_{0}^{t}\left\|\frac{\partial a}{\partial t}\left(t^{\prime}\right)\right\|_{L^{\infty}(\Omega)}^{2}\left\|u_{n}\left(t^{\prime}\right)\right\|_{H_{0}^{1}(\Omega)}^{2} d t^{\prime} .
\end{aligned}
$$

From now on, we will use in this proof the norm of $H_{0}^{1}(\Omega)$ defined by

$$
\|w\|_{H_{0}^{1}(\Omega)}^{2}=\int_{\Omega}\left|\frac{\partial w}{\partial x}\right|^{2} d x .
$$

We now assume that the Galerkin's basis $y^{1}, y^{2}, \ldots$ of $H_{0}^{1}(\Omega)$ is orthogonal in $L^{2}(\Omega)$ and is such that $y^{1}=u_{0}$, which is possible when

$$
u_{0} \in H_{0}^{1}(\Omega) .
$$


Then $u_{n}(0)=u_{0}$ for every $n$ and for every $t^{\prime}, 0 \leq t^{\prime} \leq T$

$$
u_{n}\left(t^{\prime}\right)=u_{n}(0)+\int_{0}^{t^{\prime}} \frac{\partial u_{n}}{\partial t}\left(t^{\prime \prime}\right) d t^{\prime \prime}=u_{0}+\int_{0}^{t^{\prime}} w_{n}\left(t^{\prime \prime}\right) d t^{\prime \prime} .
$$

Therefore for every $t^{\prime}, 0 \leq t^{\prime} \leq T$

$$
\left\|u_{n}\left(t^{\prime}\right)\right\|_{H_{0}^{1}(\Omega)}^{2} \leq 2\left\|u_{0}\right\|_{H_{0}^{1}(\Omega)}^{2}+2 T \int_{0}^{t^{\prime}}\left\|w_{n}\left(t^{\prime \prime}\right)\right\|_{H_{0}^{1}(\Omega)}^{2} d t^{\prime \prime},
$$

which implies that for every $t, 0 \leq t \leq T$

$$
\left\{\begin{array}{l}
\frac{c_{5}}{2} \int_{\Omega}\left|\left(w_{n}(t, x)\right)\right|^{2} d x+\frac{c_{1}}{2} \int_{0}^{t}\left\|w_{n}\left(t^{\prime}\right)\right\|_{H_{0}^{1}(\Omega)}^{2} d t^{\prime} \leq \\
\leq \frac{c_{6}}{2 c_{5}^{2}}\left\|h(0)+\frac{\partial}{\partial x}\left(a(0, x) \frac{\partial u_{0}}{\partial x}\right)\right\|_{L^{2}(\Omega)}^{2}+ \\
+\frac{1}{c_{1}}\left\|\frac{\partial h}{\partial t}\right\|_{L^{2}\left(0, T ; H^{-1}(\Omega)\right)}^{2}+\frac{2}{c_{1}}\left\|u_{0}\right\|_{H_{0}^{1}(\Omega)}^{2}\left\|\frac{\partial a}{\partial t}\right\|_{L^{2}\left(0, T ; L^{\infty}(\Omega)\right)}^{2}+ \\
+\frac{2 T}{c_{1}} \int_{0}^{t}\left\|\frac{\partial a}{\partial t}\left(t^{\prime}\right)\right\|_{L^{\infty}(\Omega)}^{2}\left(\int_{0}^{t^{\prime}}\left\|w_{n}\left(t^{\prime \prime}\right)\right\|_{H_{0}^{1}(\Omega)}^{2} d t^{\prime \prime}\right) d t^{\prime} .
\end{array}\right.
$$

Let $Z$ be the constant defined by

$$
\begin{aligned}
Z= & \frac{c_{6}}{2 c_{5}^{2}}\left\|h(0)+\frac{\partial}{\partial x}\left(a(0, x) \frac{\partial u_{0}}{\partial x}\right)\right\|_{L^{2}(\Omega)}^{2}+ \\
& +\frac{1}{c_{1}}\left\|\frac{\partial h}{\partial t}\right\|_{L^{2}\left(0, T ; H^{-1}(\Omega)\right)}^{2}+\frac{2}{c_{1}}\left\|u_{0}\right\|_{H_{0}^{1}(\Omega)}^{2}\left\|\frac{\partial a}{\partial t}\right\|_{L^{2}\left(0, T ; L^{\infty}(\Omega)\right)}^{2},
\end{aligned}
$$

and let $\varphi_{n}:(0, T) \rightarrow \mathbb{R}^{+}$be the function defined by

$$
\varphi_{n}(t)=Z+\frac{2 T}{c_{1}} \int_{0}^{t}\left\|\frac{\partial a}{\partial t}\left(t^{\prime}\right)\right\|_{L^{\infty}(\Omega)}^{2}\left(\int_{0}^{t^{\prime}}\left\|w_{n}\left(t^{\prime \prime}\right)\right\|_{H_{0}^{1}(\Omega)}^{2} d t^{\prime \prime}\right) d t^{\prime} .
$$

In view of (3.55), the function $\varphi_{n}$ satisfies for every $t, 0 \leq t \leq T$

$$
\left\{\begin{aligned}
0 \leq \varphi_{n}^{\prime}(t) & =\frac{2 T}{c_{1}}\left\|\frac{\partial a}{\partial t}(t)\right\|_{L^{\infty}(\Omega)}^{2} \int_{0}^{t}\left\|w_{n}\left(t^{\prime}\right)\right\|_{H_{0}^{1}(\Omega)}^{2} d t^{\prime} \leq \\
& \leq \frac{2 T}{c_{1}}\left\|\frac{\partial a}{\partial t}(t)\right\|_{L^{\infty}(\Omega)}^{2} \frac{2}{c_{1}} \varphi_{n}(t), \\
\varphi_{n}(0)=Z . &
\end{aligned}\right.
$$


In view of (3.49), Gronwall's inequality then implies that $\varphi_{n}$ is bounded in $L^{\infty}(0, T)$, which in turn implies using (3.55) that $w_{n}$ is bounded in $L^{\infty}\left(0, T ; L^{2}(\Omega)\right) \cap L^{2}\left(0, T ; H_{0}^{1}(\Omega)\right)$ by a constant which depends only on $c_{1}, c_{5}$, $Z$ and on $\left\|\frac{\partial a}{\partial t}\right\|_{L^{2}\left(0, T ; L^{\infty}(\Omega)\right)}$.

Since $w_{n}=\frac{\partial u_{n}}{\partial t}($ see $(3.47))$, and since $u_{n}$ tends to $u$ in $\mathscr{D}^{\prime}(Q)$ (see (3.46)), this proof implies that when (3.48), (3.49), (3.52) and (3.54) hold true, the solution $u$ of (3.42) satisfies

$$
\frac{\partial u}{\partial t} \in L^{\infty}\left(0, T ; L^{2}(\Omega)\right) \cap L^{2}\left(0, T ; H_{0}^{1}(\Omega)\right),
$$

with

$$
\left\|\frac{\partial u}{\partial t}\right\|_{L^{\infty}\left(0, T ; L^{2}(\Omega)\right)}+\left\|\frac{\partial u}{\partial t}\right\|_{L^{2}\left(0, T ; H_{0}^{1}(\Omega)\right)} \leq F^{\star \star}
$$

where $F^{\star \star}$ depends only on $c_{1}, c_{5}, Z$ and on $\left\|\frac{\partial a}{\partial t}\right\|_{L^{2}\left(0, T ; L^{\infty}(\Omega)\right)}$.

\section{Third step: end of the proof}

We now apply the previous abstract result to every function $u$ such that $(u, \tau)$ satisfies (2.61)-(2.66). We have to check that (3.48), (3.49), (3.52) and (3.54) hold true.

Condition (3.48) follows from the definition (3.44) of $h$, from hypotheses (3.31) and (3.32) on $f, v_{a}$ and $v_{b}$, from $\frac{\partial \bar{v}}{\partial x \partial t} \in L^{2}(\Omega)$ (which follows from (2.43) and (2.9)), and finally from $\frac{\partial \bar{v}}{\partial x} \in L^{\infty}(Q)$ (which again follows from (2.43) and (2.9)) and from the regularity

$$
\frac{\partial a}{\partial t} \in L^{2}(Q)
$$

where the latest regularity, which is by no means straightforward, is obtained in the following way. By the definition (3.43) of $a$, the equation (2.64) on $\tau$ and the definition (2.67) of $\sigma$, one has

$$
\frac{\partial a}{\partial t}=\frac{\partial \lambda}{\partial r}(x, \tau) \frac{\partial \tau}{\partial t}=\frac{\partial \lambda}{\partial r}(x, \tau) \sigma^{2}
$$

But when $(u, \tau)$ is a solution of (2.61)-(2.66), then the stress $\sigma$ satisfies (2.68), i.e.

$$
\sigma \in L^{\infty}\left(0, T ; L^{2}(\Omega)\right) \cap L^{2}\left(0, T ; H^{1}(\Omega)\right),
$$

and therefore the result (3.70) of Lemma 3.1 below implies that

$$
\sigma \in L^{4}\left(0, T ; L^{\infty}(\Omega)\right)
$$


which combined with (3.57) and the Lipschitz continuity (3.13) of $\lambda$ implies that $\frac{\partial a}{\partial t}$ belongs to $L^{2}\left(0, T ; L^{\infty}(\Omega)\right)$, hence in particular that (3.56) holds true. Here we have used in a crucial way the fact that $\Omega$ is one dimensional.

Condition (3.49) is stronger than (3.56), but has just been proved above.

Condition (3.52) follows from the definitions (3.44), (3.43), (2.43) and (2.44) of $h, a, \bar{v}$ and $u_{0}$, from the compatibility condition (3.33) and from the fact that $\rho(x) \frac{\partial \bar{v}}{\partial t}(0, x) \in L^{2}(\Omega)$, which follows from (2.43), (2.6) and (3.32).

Finally condition (3.54) is nothing but (2.46), which immediately follows from (2.44), (2.9), (2.10) and (2.12).

This completes the proof of Theorem 3.2, since the a priori estimate (3.35) then follows from the a priori estimate obtained in the above (abstract) step.

Observe that in the (correct) proof made above in the second and third steps, we used the $L^{4}\left(0, T ; L^{\infty}(\Omega)\right)$ regularity of $\sigma$, while in the formal proof made above in the first step, we used the $L^{6}(Q)$ regularity of $\sigma$. These regularities, which follow from the interpolation results (3.70) and (3.69) of Lemma 3.1, are both specific to the case where $\Omega$ is one dimensional.

\subsection{Final result}

From the existence Theorem 2.1, the uniqueness Theorem 3.1 and the regularity Theorem 3.2, we deduce in particular the following result.

\section{Theorem 3.3 (Existence, uniqueness and regularity) Assume that}

$$
\begin{gathered}
f \in L^{\infty}\left(0, T ; L^{2}(\Omega)\right), \\
\theta_{0} \in L^{\infty}(\Omega),
\end{gathered}
$$

and that hypotheses (2.1)-(2.12), (3.1) and (3.31)-(3.33) hold true. Then there exists a unique couple $(v, \theta)$ which satisfies (2.13)-(2.19). This couple depends on the data in a locally Lipschitz continuous way (see (3.2)). Moreover $v, \theta$ and $\sigma$ defined by (2.20) enjoy in particular the following regularities

$$
\begin{gathered}
v \in L^{\infty}(Q) \cap H^{1}(Q), \\
\theta \in L^{\infty}(Q), \\
\sigma \in L^{\infty}(Q) \cap H^{1}(Q), \\
\frac{\partial v}{\partial x} \in L^{\infty}(Q) .
\end{gathered}
$$

As far as Neumann's and mixed boundary conditions are concerned, results similar to Theorem 3.3 hold true in the frameworks of Theorems 2.1bis and 2.1ter. We leave their statements and proofs to the reader.

As announced in the Introduction, Theorem 3.3 ensures the existence and uniqueness of a solution of (1.1)-(1.5) with $v, \theta$ and $\sigma$ in $L^{\infty}(Q)$. 
Actually $v, \theta, \sigma, \frac{\partial v}{\partial x}$ and $\frac{\partial v}{\partial t}$ enjoy additional regularity properties which we did not list in Theorem 3.3 for the sake of simplicity, but which can be deduced from the results of Theorem 3.2, Proposition 3.1, Lemma 3.1 and straightforward consequences of them. In particular under hypotheses of Theorem 3.3, $\frac{\partial v}{\partial t}$ satisfies

$$
\left\{\begin{array}{r}
\frac{\partial v}{\partial t} \in L^{\infty}\left(0, T ; L^{2}(\Omega)\right) \cap L^{2}\left(0, T ; H^{1}(\Omega)\right) \cap \\
\cap L^{4}\left(0, T ; L^{\infty}(\Omega)\right) \cap L^{6}\left(0, T ; L^{6}(\Omega)\right),
\end{array}\right.
$$

since this regularity immediately follows from (3.34) and from Lemma 3.1 below because $\Omega$ is one dimensional.

Moreover under the hypotheses of Theorem 3.3, the transformed temperature $\tau$ defined by (2.58) enjoys additional regularity properties. In particular if one further assumes that

$$
f \in L^{2}\left(0, T ; L^{\infty}(\Omega)\right)
$$

one obtains that

$$
\tau \in W^{1, \infty}(Q) .
$$

Indeed this regularity easily follows from the problem (3.12) satisfied by $\tau$, i.e.

$$
\frac{\partial \tau}{\partial t}=\sigma^{2} \quad \text { in } \mathscr{D}^{\prime}(Q), \quad \tau(0, x)=0 \quad \text { a.e. } x \in \Omega,
$$

which combined with $L^{\infty}(Q)$ regularity (3.64) of $\sigma$ imply that $\frac{\partial \tau}{\partial t}$ and $\tau$ belong to $L^{\infty}(Q)$. On the other hand, differentiation of (3.12) with respect to $x$ yields

$$
\frac{\partial}{\partial t}\left(\frac{\partial \tau}{\partial x}\right)=2 \sigma \frac{\partial \sigma}{\partial x} \quad \text { in } \mathscr{D}^{\prime}(Q), \quad \frac{\partial \tau}{\partial x}(0, x)=0 \quad \text { a.e. in } \Omega .
$$

The $L^{\infty}(Q)$ regularity (3.64) of $\sigma$ and the $L^{2}\left(0, T ; L^{\infty}(\Omega)\right)$ regularity of $\frac{\partial \sigma}{\partial x}$, which follows from the equation

$$
\frac{\partial \sigma}{\partial x}=f-\rho \frac{\partial v}{\partial t} \quad \text { in } \mathscr{D}^{\prime}(Q)
$$

from hypotheses (3.67) on $f$ (this is the only point where this new hypothesis is used) and from the regularity $L^{2}\left(0, T ; L^{\infty}(\Omega)\right)$ of $\frac{\partial v}{\partial t}$ (which follows from (3.34) and from $H^{1}(\Omega) \subset L^{\infty}(\Omega)$ ), then imply that $\frac{\partial}{\partial t}\left(\frac{\partial \tau}{\partial x}\right)$ belongs to $L^{2}\left(0, T ; L^{\infty}(\Omega)\right)$, which with the initial condition $\frac{\partial \tau}{\partial x}(0, x)=0$ yields $\frac{\partial \tau}{\partial x} \in L^{\infty}(Q)$ and completes the proof of (3.68). 
Observe however, similarly to the comment made after the statement of Proposition 3.1 (straightforward regularity of $\tau$ ), that regularity (3.68) cannot be obtained on $\theta$ as far as regularity in $x$ is concerned. Consider indeed for example the simple case where

$$
c(x, s) \mu(x, s)= \begin{cases}m_{a}(s) & \text { if } a \leq x<\frac{a+b}{2}, \\ m_{b}(s) & \text { if } \frac{a+b}{2}<x \leq b,\end{cases}
$$

and where $\theta_{0}=0$. Then the relation (2.58) between $\tau$ and $\theta$ reads as

$$
\tau(t, x)= \begin{cases}M_{a}(\theta(t, x)) & \text { if } a \leq x<\frac{a+b}{2}, \\ M_{b}(\theta(t, x)) & \text { if } \frac{a+b}{2}<x \leq b,\end{cases}
$$

where in view of (2.52) the functions $M_{a}$ and $M_{b}$ are given by

$$
M_{a}(s)=\int_{0}^{s} m_{a}\left(s^{\prime}\right) d s^{\prime}, \quad M_{b}(s)=\int_{0}^{s} m_{b}\left(s^{\prime}\right) d s^{\prime} .
$$

This prevents $\theta$ to be continuous (and a fortiori Lipschitz continuous) in $x$ on the line $x=\frac{a+b}{2}$ when $\tau$ is Lipschitz continuous on $Q$.

Proof of Theorem 3.3 The proof is easy and follows from the results obtained in Theorems 2.1, 3.1 and 3.2.

Existence and uniqueness have already been proved in Theorems 2.1 and 3.1. The regularity (3.62) of $v$ follows from (2.13) since $H^{1}(\Omega) \subset L^{\infty}(\Omega)$ in the one dimensional case.

For what concerns the regularity of $\sigma$, we already know from (2.21) that $\sigma$ and $\frac{\partial \sigma}{\partial x}$ belongs to $L^{2}(Q)$. On the other hand

$$
\begin{aligned}
\frac{\partial \sigma}{\partial t} & =\mu(x, \theta) \frac{\partial^{2} v}{\partial x \partial t}+\frac{\partial \mu}{\partial s}(x, \theta) \frac{\partial \theta}{\partial t} \frac{\partial v}{\partial x}= \\
& =\mu(x, \theta) \frac{\partial^{2} v}{\partial x \partial t}+\frac{\partial \mu}{\partial s}(x, \theta) \frac{1}{c(x, \theta) \mu(x, \theta)} \sigma^{2} \frac{1}{\mu(x, \theta)} \sigma .
\end{aligned}
$$

Since $\frac{\partial^{2} v}{\partial x \partial t} \in L^{2}(Q)$ by (3.34), and since $\frac{\partial \mu}{\partial s}$ is bounded by hypothesis (3.1), while $\sigma \in L^{6}(Q)$ by (2.21) and the interpolation result (3.69) of Lemma 3.1 below, we have $\frac{\partial \sigma}{\partial t} \in L^{2}(Q)$. This completes the proof of the fact that $\sigma \in H^{1}(Q)$. Finally equation (2.15), which reads as

$$
\frac{\partial \sigma}{\partial x}=\rho(x) \frac{\partial v}{\partial t}-f \quad \text { in } \mathscr{D}^{\prime}(Q)
$$


together with hypothesis (3.60) on $f$ (this is the only point where this hypothesis is used) and the regularity (3.34) on $\frac{\partial v}{\partial t}$, implies that $\frac{\partial \sigma}{\partial x}$ belongs to $L^{\infty}\left(0, T ; L^{2}(\Omega)\right)$. But $\sigma$ also belongs to $L^{\infty}\left(0, T ; L^{2}(\Omega)\right)$ (see $\left.(2.21)\right)$, and therefore $\sigma$ belongs to $L^{\infty}\left(0, T ; H^{1}(\Omega)\right) \subset L^{\infty}\left(0, T ; L^{\infty}(\Omega)\right)$. This completes the proof of the regularity (3.64) of $\sigma$, and immediately implies the $L^{\infty}(Q)$ regularity (3.65) of $\frac{\partial v}{\partial x}$ since $\frac{\partial v}{\partial x}=\frac{1}{\mu(x, \theta)} \sigma$.

The $L^{\infty}(Q)$ regularity (3.63) of $\theta$ finally follows from the fact that $\sigma$ belongs to $L^{2}\left(0, T ; H^{1}(\Omega)\right) \subset L^{2}\left(0, T ; L^{\infty}(\Omega)\right)$ (see $\left.(2.21)\right)$ and from the equation

$$
\frac{\partial \theta}{\partial t}=\frac{1}{c(x, \theta) \mu(x, \theta)} \sigma^{2},
$$

which together imply that $\frac{\partial \theta}{\partial t} \in L^{1}\left(0, T ; L^{\infty}(\Omega)\right)$, and from the $L^{\infty}(\Omega)$ hypothesis (3.61) on $\theta_{0}$ (this is the only point where this hypothesis is used).

This completes the proof of Theorem 3.3.

\subsection{A one dimensional interpolation lemma}

This short Subsection is devoted to the statement and proof of two interpolation results, which are valid only in the case where $\Omega$ is one dimensional.

Lemma 3.1 (Interpolation) When $\Omega=(a, b) \subset \mathbb{R}$, one has

$$
\begin{aligned}
& L^{\infty}\left(0, T ; L^{2}(\Omega)\right) \cap L^{2}\left(0, T ; H^{1}(\Omega)\right) \subset L^{6}\left(0, T ; L^{6}(\Omega)\right), \\
& L^{\infty}\left(0, T ; L^{2}(\Omega)\right) \cap L^{2}\left(0, T ; H^{1}(\Omega)\right) \subset L^{4}\left(0, T ; L^{\infty}(\Omega)\right) .
\end{aligned}
$$

Proof By interpolation, one has

$$
\|\psi\|_{H^{1 / 3}(\Omega)} \leq C\|\psi\|_{L^{2}(\Omega)}^{2 / 3}\|\psi\|_{H^{1}(\Omega)}^{1 / 3} \quad \forall \psi \in H^{1}(\Omega),
$$

for a constant $C$ which depends on $(b-a)$. On the other hand, Sobolev's embedding theorem $H^{1 / 3}(\Omega) \subset L^{6}(\Omega)$, which holds since $\frac{1}{6}=\frac{1}{2}-\frac{\frac{1}{3}}{1}$, asserts that

$$
\|\psi\|_{L^{6}(\Omega)} \leq K\|\psi\|_{H^{1 / 3}(\Omega)} \quad \forall \psi \in H^{1 / 3}(\Omega),
$$

for an absolute constant $K$. Note that the latest result is specific to the one dimensional case.

Therefore for every $\sigma \in L^{\infty}\left(0, T ; L^{2}(\Omega)\right) \cap L^{2}\left(0, T ; H^{1}(\Omega)\right)$, one has

$$
\begin{aligned}
\|\sigma\|_{L^{6}\left(0, T ; L^{6}(\Omega)\right)}^{6} & =\int_{0}^{T}\|\sigma(t)\|_{L^{6}(\Omega)}^{6} d t \leq K^{6} \int_{0}^{T}\|\sigma(t)\|_{H^{1 / 3}(\Omega)}^{6} d t \leq \\
& \leq K^{6} \int_{0}^{T} C^{6}\|\sigma(t)\|_{L^{2}(\Omega)}^{4}\|\sigma(t)\|_{H^{1}(\Omega)}^{2} d t \leq \\
& \leq K^{6} C^{6}\|\sigma\|_{L^{\infty}\left(0, T ; L^{2}(\Omega)\right)}^{4}\|\sigma\|_{L^{2}\left(0, T ; H^{1}(\Omega)\right)}^{2}
\end{aligned}
$$

which proves (3.69). 
Let us now prove (3.70). Since $\Omega$ is one dimensional, the interpolation result $\left(H^{1}(\Omega), L^{2}(\Omega)\right)_{\frac{1}{2}, 1} \subset L^{\infty}(\Omega)$ implies that

$$
\|\psi\|_{L^{\infty}(\Omega)} \leq C\|\psi\|_{L^{2}(\Omega)}^{1 / 2}\|\psi\|_{H^{1}(\Omega)}^{1 / 2} \quad \forall \psi \in H^{1}(\Omega),
$$

for a constant $C$ which depends on $(b-a)$. This result is specific to the one dimensional case and can be proved in an elementary way as follows. For every $x$ and $y$ with $a<x<y<b$, one has using Cauchy-Schwartz's inequality

$$
\begin{aligned}
\psi^{2}(y) & =\psi^{2}(x)+\int_{x}^{y} 2 \psi(x) \frac{d \psi}{d x}(s) d s \leq \\
& \leq \psi^{2}(x)+2 \int_{a}^{b}|\psi(s)|\left|\frac{d \psi}{d x}(s)\right| d s \leq \\
& \leq \psi^{2}(x)+2\|\psi\|_{L^{2}(\Omega)}\left\|\frac{d \psi}{d x}\right\|_{L^{2}(\Omega)} .
\end{aligned}
$$

Integrating in $x$ on $\Omega$ yields

$$
\begin{aligned}
\psi^{2}(y) & \leq \frac{1}{b-a}\|\psi\|_{L^{2}(\Omega)}^{2}+2\|\psi\|_{L^{2}(\Omega)}\left\|\frac{d \psi}{d x}\right\|_{L^{2}(\Omega)}= \\
& =\|\psi\|_{L^{2}(\Omega)}\left(\frac{1}{b-a}\|\psi\|_{L^{2}(\Omega)}+2\left\|\frac{d \psi}{d x}\right\|_{L^{2}(\Omega)}\right)
\end{aligned}
$$

which immediately implies (3.71).

Therefore for every $\sigma \in L^{\infty}\left(0, T ; L^{2}(\Omega)\right) \cap L^{2}\left(0, T ; H^{1}(\Omega)\right)$, one has

$$
\begin{aligned}
\|\sigma\|_{L^{4}\left(0, T ; L^{\infty}(\Omega)\right)}^{4} & =\int_{0}^{T}\|\sigma(t)\|_{L^{\infty}(\Omega)}^{4} d t \leq \\
& \leq C^{4} \int_{0}^{T}\|\sigma(t)\|_{L^{2}(\Omega)}^{2}\|\sigma(t)\|_{H^{1}(\Omega)}^{2} d t \leq \\
& \leq C^{4}\|\sigma\|_{L^{\infty}\left(0, T ; L^{2}(\Omega)\right)}^{2}\|\sigma\|_{L^{2}\left(0, T ; H^{1}(\Omega)\right)}^{2}
\end{aligned}
$$

which proves (3.70).

This completes the proof of Lemma 3.1.

Acknowledgements The authors warmly thank Luc Tartar for interesting discussions and for his friendly help. Part of the results of the present work have been obtained while the first author was visiting the Laboratoire Jacques-Louis Lions (at that time called Laboratoire d'analyse numérique) of the Université Pierre et Marie Curie (Paris VI) during his sabbatical in 198889 , but they were not summitted for publication immediately. The support and hospitality of the Laboratoire Jacques-Louis Lions are gratefully acknowledged. The support of the European RTD project Lifebelt IST-2001-38165 is also gratefully acknowledged. Finally the interest and support of UIK and UMT are sincerely acknowledged. 


\section{References}

1. Charalambakis, N.: Adiabatic shearing flow caused by time-dependent inertial force. Quart. Appl. Math. 42, 275-280 (1984)

2. Charalambakis, N., Murat, F.: Weak solutions to initial boundary value problems for the shearing of nonhomogeneous thermoviscoplastic materials. Proc. Royal Soc. Edinburgh 113A, 257-265 (1989)

3. Charalambakis, N., Murat, F.: Homogenization of stratified thermoviscoplastic materials. Quart. Appl. Math. 64, 359-399 (2006)

4. Dafermos, C.M., Hsiao, L.: Adiabatic shearing of incompressible fluids with temperature dependent viscosities. Quart. Appl. Math. 41, 45-58 (1983)

5. Hodowany, J., Ravichandran, G., Rosakis, A.J., Rosakis, P.: Partition of plastic work into heat and stored energy in metals. J. Exp. Mech. 40, 113-123 (2000)

6. Lemaitre, J., Chaboche, J.-L.: Mécanique des matériaux solides. Dunod, Paris (2001)

7. Molinari, A., Clifton, R.: Analytical characterization of shear localization. J. Appl. Mech. 54, 806-912 (1987)

8. Rosakis, P., Rosakis, A.J., Ravichandran, G., Hodowany, J.: A thermodynamical internal variable model for the partition of plastic work into heat and stored energy in metals. J. Mech. Phys. Solids 48, 582-607 (2000)

9. Tzavaras, A.: Shearing of materials exhibiting thermal softening or temperature dependent viscosity. Quart. Appl. Math. 44, 1-12 (1986)

10. Tzavaras, A.: Plastic shearing of materials exhibiting strain hardening or strain softening. Arch. Rat. Mech. Anal. 99, 39-58 (1986)

11. Wright, T.W.: The physics and mathematics of adiabatic shear bands. Cambridge University Press, Cambridge (2002) 